\title{
Large-eddy simulation and wall modelling of turbulent channel flow
}

\author{
D. CHUNG $\dagger$ AND D. I. PULLIN \\ Graduate Aeronautical Laboratories, California Institute of Technology, Pasadena, CA 91125, USA
}

(Received 28 May 2008 and in revised form 8 March 2009)

We report large-eddy simulation (LES) of turbulent channel flow. This LES neither resolves nor partially resolves the near-wall region. Instead, we develop a special near-wall subgrid-scale (SGS) model based on wall-parallel filtering and wall-normal averaging of the streamwise momentum equation, with an assumption of local inner scaling used to reduce the unsteady term. This gives an ordinary differential equation (ODE) for the wall shear stress at every wall location that is coupled with the LES. An extended form of the stretched-vortex SGS model, which incorporates the production of near-wall Reynolds shear stress due to the winding of streamwise momentum by near-wall attached SGS vortices, then provides a $\log$ relation for the streamwise velocity at the top boundary of the near-wall averaged domain. This allows calculation of an instantaneous slip velocity that is then used as a 'virtual-wall' boundary condition for the LES. A Kármán-like constant is calculated dynamically as part of the LES. With this closure we perform LES of turbulent channel flow for Reynolds numbers $R e_{\tau}$ based on the friction velocity $u_{\tau}$ and the channel half-width $\delta$ in the range $2 \times 10^{3}$ to $2 \times 10^{7}$. Results, including SGS-extended longitudinal spectra, compare favourably with the direct numerical simulation (DNS) data of Hoyas \& Jiménez (2006) at $R e_{\tau}=2003$ and maintain an $O(1)$ grid dependence on $R e_{\tau}$.

\section{Introduction}

Large-eddy simulation (LES), the numerical simulation of fluid flow in which large-scale motion ('large eddy') is computed directly while small-scale motion is modelled, shows considerable potential for the under-resolved but accurate simulation of complex turbulent flows. To date, an LES practitioner wishing to simulate a turbulent flow effectively assumes that the dynamics of large-scale, resolved eddies are dominated by the flow geometry along with associated large-scale boundary conditions or other turbulence-generating forcing. Accordingly, it is then thought sufficient to simulate numerically only those large eddies yet retain the capability to accurately recover at least first- and second-order statistics (means, correlations and power spectral densities). The underlying ansatz is that the small scales are universal and can be parameterized in some way by the local resolved flow properties, with no explicit dependence on boundary conditions. If these assumptions are valid, successful LES should require only a computational grid that scales with flow geometry; specifically, the computational grid should be independent, $O(1)$ or weakly dependent, $O(\log R e)$,

$\dagger$ Email address for correspondence: dchung@ caltech.edu 
say, on the Reynolds number Re. LES promises enormous resource savings when compared to the prohibitively expensive but accurate direct numerical simulation (DNS), where all scales of motion are computed. Typically, the required number of grid points for DNS scales as $O\left(R e^{9 / 4}\right)$, which measures the size of the largest eddy relative to the smallest eddy in three dimensions. Further, the need to compute all scales and then to perform massive data reduction renders DNS an inefficient and unpractical engineering design tool.

Since the early work on LES by Smagorinsky (1963) and Deardorff (1970), LES has met with a mix of success and challenges. It is fair to say that the outcome of an LES depends largely on the validity of the assumptions held by our hypothetical LES practitioner. For flows in which these assumptions apply, typically unbounded flows, such as homogeneous isotropic turbulence (e.g. Misra \& Pullin 1997), shear and wake/jet turbulence and even the more challenging Richtmyer-Meshkov instability (e.g. Hill, Pantano \& Pullin 2006), LES has performed exceedingly well. Despite continuing efforts, however, (e.g. Cabot \& Moin 1999; Piomelli \& Balaras 2002; Wang \& Moin 2002; Templeton, Medic \& Kalitzin 2005; Piomelli 2008), the LES of wall-bounded flows, while improving, remains a challenging area of research.

Near walls, 'large eddies', implicitly defined by the coarse grid, simply do not exist (Pope 2004). The near-wall eddies, having sizes constrained by the wall, become under-resolved at high $R e$ and are therefore considered part of the subgrid motion. In unbounded flows, the large eddies, carrying most of the turbulent kinetic energy, set the length and time scales that describe the rest of the small-scale turbulence. This picture is reversed near the wall, where the most energetically productive eddies are necessarily part of the small-scale motion. Further, both these descriptions are present in wall-bounded flows, and both contribute significantly to the overall turbulent flow field. This was demonstrated by Hutchins \& Marusic (2007), who plotted velocity spectra of high-Re boundary layers at various wall distances and showed the existence of two distinct energetic peaks: one that scales with viscous units, another that scales with boundary layer thickness. A related complication is that, given sufficiently high $R e$, even the mean velocity gradient is too steep to be resolved on the coarse LES grid, appearing as a numerical discontinuity. The jump conditions across this discontinuity depend on unclosed turbulent stresses, which themselves require reliable models.

One can always perform a partially resolved LES, where the near-wall grid is refined to resolve the near-wall eddies (e.g. Voelkl, Pullin \& Chan 2000). Although this kind of simulation has its role in the development of LES models, its resolution requirement, which scales as $O\left(R e^{1.8}\right)$ (Piomelli 2008), is almost as restrictive as DNS. But if we require that the resolution requirements of LES be at most weakly dependent on $R e$, we are forced to explicitly model the vigorous near-wall fluctuations and the numerical discontinuity (slip) in the mean velocity profile. Ample experimental evidence that suggests some degree of universality in wall-bounded flows, e.g.the empirical log law of the wall, offers hope that a viable LES wall model could be found.

Past modelling efforts were focused on fitting a log law to the near-wall filtered velocity to obtain the implied wall shear stress, which, in turn, is used as a wall stress boundary condition (Cabot \& Moin 1999; Pantano et al. 2008). Instead of the log law, the thin boundary layer equations (TBLEs) coupled with a damped mixinglength eddy viscosity can also be used, giving good agreement with experiments even in separating flows (Cabot \& Moin 1999; Wang \& Moin 2002). Yet another method of determining the wall stress is by matching the LES eddy viscosity with the 
Reynolds-averaged Navier-Stokes (RANS) eddy viscosity (Templeton et al. 2005), with success at high $R e$.

In this paper we apply the wall stress boundary condition in reverse: given the wall shear stress, we impose the corresponding slip velocity at a predetermined lifted virtual boundary, located well above the viscous layer and fixed in outer coordinates. We approach the wall modelling problem by considering the physics of near-wall vortices, giving us a relationship between the wall shear stress and slip velocity. We assume that the dynamics of each wall-adjacent cell are partially determined by its wall shear stress, similar to the class of equilibrium-stress models (see Piomelli 2008), but, presently, the wall shear stress is governed by its own ordinary differential equation (ODE) derived from assumptions somewhat like those used to derive the TBLE. We do not, however, use an inner mesh to solve the TBLE, called a zonal approach by Piomelli \& Balaras (2002); instead, we assume only that the law of the wall, irrespective of its detailed form, holds locally. This is then used only for the treatment of one term in our reduction of the streamwise momentum equation. Indeed, our approach attempts to essentially eliminate the Reynolds number dependence of the wall model. In terms of the classification of Piomelli (2008), we have combined elements of both the equilibrium-stress models and the zonal approaches, since we assume a localized near-wall layer characterized by its wall shear stress, and we also solve a reduced form of the TBLE.

Much understanding of wall turbulence has been gained by studying simple physical models based on a statistical ensemble of vortical structures that have tractable analytical expressions. The attached eddy model of Townsend (1976; see also Perry \& Chong 1982; Nickels et al. 2007) was able to reproduce various observed physics of wall turbulence, e.g.the mean velocity profile, the Reynolds stress profiles and some aspects of the velocity spectra. A part of our wall model is based on related ideas, where we assume a hierarchy of vortices with sizes that scale with wall distance. These are aligned in the streamwise direction and act to wrap the streamwise momentum, that is the vortex axial velocity, as if it behaved locally like a passive scalar.

In what follows, the filtered Navier-Stokes equations are given in $\S 2.1$, and the basics of the stretched-vortex subgrid-scale (SGS) model (Misra \& Pullin 1997) are described in $\S 2.2$. This is followed by the development of an extended SGS model that incorporates the effect of vortex winding of axial velocity to produce off-diagonal components of the vortex-frame subgrid Reynolds stresses $(\S 2.3)$. We then introduce the ideas of wall-normal averaging (\$3.1) and local inner scaling (§3.2), which together lead to an ODE for the wall-normal streamwise velocity gradient at each wall-adjacent cell without the need to resolve the wall layer. The SGS dynamics in the wall-adjacent cell are split into three layers $(\$ 3.3)$ : a viscous dominated region, a constant-stress region and an outer region. The extended SGS model, applied within the constantstress layer with streamwise-aligned SGS vortices, leads to a log relationship for the streamwise velocity. A factor, equivalent to an inverse Kármán-like constant, that multiplies the $\log$ term is calculated dynamically by the LES to within a mixing constant. The latter is determined a priori by a stress-matching argument. Together with the use of an empirically determined matching point, in inner variables, for the logarithmic and viscous sublayer velocity intersection, the overall SGS wall model provides a slip boundary condition for the streamwise velocity at a lifted 'virtual' wall (§3.4) that is placed within the log layer. Statistical estimates of the Kármán 'constant' can then be obtained from the LES. The results obtained from LES of channel flow are described in detail in $\S 4$, and details of the numerical method are in Appendix A. 


\section{Equations of motion and SGS model}

\subsection{Filtered Navier-Stokes equations}

In this paper, the following notation is adopted: $x_{1}$ or $x$ is the streamwise coordinate; $x_{2}$ or $y$ is the spanwise coordinate; and $x_{3}$ or $z$ is the wall-normal coordinate. We obtain the governing equations for LES by applying a filter to the Navier-Stokes equations. Presently this filtering operation is considered strictly formal; no explicit filtering is used in the present work. We shall denote with a tilde any field quantity $\phi(x)$ subjected to the filter $G$ with cutoff length $\Delta_{c}$, that is

$$
\widetilde{\phi}(\boldsymbol{x})=\int G\left(\boldsymbol{x}-\boldsymbol{x}^{\prime} ; \Delta_{c}\right) \phi\left(\boldsymbol{x}^{\prime}\right) \mathrm{d} \boldsymbol{x}^{\prime} .
$$

Except for $\Delta_{c}$, which is prescribed through the SGS model, no other filter properties enter into the flow simulation. After applying the filter and explicitly decomposing the velocity field as $u_{i} \equiv \widetilde{u}_{i}+u_{i}^{\prime}$, we obtain the filtered Navier-Stokes equations

$$
\begin{aligned}
\frac{\partial \widetilde{u}_{i}}{\partial t}+\frac{\partial \widetilde{u}_{i} \widetilde{u}_{j}}{\partial x_{j}} & =-\frac{\partial \widetilde{p}}{\partial x_{i}}+v \frac{\partial^{2} \widetilde{u}_{i}}{\partial x_{j}^{2}}-\frac{\partial T_{i j}}{\partial x_{j}}+f(t) \delta_{i 1}, \quad \frac{\partial \widetilde{u}_{i}}{\partial x_{i}}=0, \\
T_{i j} & =\widetilde{u_{i} u_{j}}-\widetilde{u}_{i} \widetilde{u}_{j}=\widetilde{\widetilde{u}_{i} u_{j}^{\prime}}+\widetilde{u_{i}^{\prime} \widetilde{u}_{j}}+\widetilde{u_{i}^{\prime} u_{j}^{\prime}} .
\end{aligned}
$$

In the above equation $T_{i j}$ is the subgrid stress tensor. We have neglected the Leonard stresses, since a numerical method with high spectral resolution is used, allowing us to focus on LES modelling; $v$ is the kinematic viscosity; $p$ is the kinematic pressure; and $f(t)$ is a force that maintains a given mass flux through the channel. Temporal filtering is implicit in LES, since the time step size is chosen by the CourantFriedrichs-Levy (CFL) condition, which is based on the advection time scale of spatially resolved scales. In other words, spatially unresolved scales are also temporally unresolved.

\subsection{The stretched-vortex $S G S$ model}

The stretched-spiral vortex (Lundgren 1982) is a physical model for turbulent fine scales, where the flow is composed of tube-like structures with concentrated vorticity. On a segment of such tubes, the flow is approximated by an axially stretched twodimensional flow. These simplified equations admit analytical large-time asymptotic solutions, from which ensemble statistics, such as correlation and spectra, follow. These have been studied extensively and were found to be consistent with experimental data (see e.g. Pullin \& Saffman 1993; Pullin \& Lundgren 2001; O’Gorman \& Pullin 2003).

Misra \& Pullin (1997) used a stretched vortex to model subgrid scales for LES in the following way: Embedded in each computational cell it is assumed that there exists a superposition of stretched vortices, each having orientation taken from a probability density function (p.d.f.). If we further assume that the subgrid ensemble dynamics are dominated by a vortex aligned with the unit vector $\boldsymbol{e}^{v}$, modelled via a delta-function p.d.f., the resulting subgrid stress tensor of the ensemble is (Misra \& Pullin 1997)

$$
T_{i j}=\left(\delta_{i j}-e_{i}^{v} e_{j}^{v}\right) K,
$$

where $K$ is the subgrid kinetic energy,

$$
K=\int_{k_{c}}^{\infty} E(k) \mathrm{d} k,
$$


where presently, we use the cutoff wavenumber $k_{c}=\pi / \Delta_{c}, \Delta_{c}=\left(\Delta_{x} \Delta_{y} \Delta_{z}\right)^{1 / 3}$, and $E(k)$ is the SGS energy spectrum.

The above expression is essentially kinematical and is independent of the detailed subgrid vortex dynamics. If it is further assumed that the SGS vortices are of the stretched-spiral type, which have energy spectra, determined by detailed Navier-Stokes dynamics, of the form (Lundgren 1982)

$$
E(k)=\mathscr{K}_{0} \epsilon^{2 / 3} k^{-5 / 3} \exp \left[-2 k^{2} v /(3|\widetilde{a}|)\right],
$$

where $\widetilde{a}=e_{i}^{v} e_{j}^{v} \widetilde{S}_{i j}$, the stretching felt along the subgrid vortex axis imposed by the resolved scales, and $\widetilde{S}_{i j}=(1 / 2)\left(\partial \widetilde{u}_{i} / \partial x_{j}+\partial \widetilde{u}_{j} / \partial x_{i}\right)$, the resolved strain-rate tensor; then combining (2.5) and (2.6), we obtain

$$
K=\frac{1}{2} \mathscr{K}_{0}^{\prime} \Gamma\left[-1 / 3, \kappa_{c}^{2}\right],
$$

where

$$
\mathscr{K}_{0}^{\prime}=\mathscr{K}_{0} \epsilon^{2 / 3} \lambda_{v}^{2 / 3}, \quad \lambda_{v}=(2 v / 3|\widetilde{a}|)^{1 / 2}, \quad \kappa_{c}=k_{c} \lambda_{v} .
$$

In the above expressions $\Gamma$ is an incomplete gamma function, and $\kappa_{c}^{2}$ can be interpreted as the inverse grid-level $R e$ : the LES model is most active when $\kappa_{c} \rightarrow 0$ and turns off for $\kappa_{c} \rightarrow \infty$.

Except for the choice of $\boldsymbol{e}^{v}$, we obtain a parameter-free SGS model by calculating the grouped constant $\mathscr{K}_{0}^{\prime}$ from the following matching procedure (Voelkl et al. 2000): Calculate the local average, denoted by \langle\rangle , of the resolved-scale second-order structure function from the running LES simulation, and match it to the stretched-spiral vortex prediction of the same grouped constant,

$$
\left\langle F_{2}\right\rangle=\left\langle 4 \int_{0}^{k_{c}} E(k)\left[1-J_{0}(k r)\right] \mathrm{d} k\right\rangle,
$$

where $F_{2}$ is the local second-order structure function as calculated from the running simulation,

$$
F_{2}=\left[\delta \widetilde{u}_{i}\right]^{2}=\left[\widetilde{u}_{i}(\boldsymbol{x})-\widetilde{u}_{i}\left(\boldsymbol{x}^{\prime}\right)\right]^{2}, \quad r^{2}=(|\delta \boldsymbol{x}|)^{2}-\left(\delta \boldsymbol{x} \cdot \boldsymbol{e}^{v}\right)^{2}, \quad \delta \boldsymbol{x}=\boldsymbol{x}-\boldsymbol{x}^{\prime} .
$$

Here, $\boldsymbol{x}^{\prime}$ is the integration variable; $J_{0}$ is the zeroth-order Bessel function of the first kind; and $r$ is the distance from $\boldsymbol{x}^{\prime}$ to the vortex axis. Substituting the energy spectrum, (2.6), and simplifying, we find that

$$
\mathscr{K}_{0}^{\prime}=\left\langle F_{2}\right\rangle /\left\langle Q\left(\kappa_{c}, d\right)\right\rangle,
$$

where

$$
d=r / \Delta_{c}, \quad Q\left(\kappa_{c}, d\right)=4 \int_{0}^{\kappa_{c}} \kappa^{-5 / 3} \mathrm{e}^{-\kappa^{2}}\left[1-J_{0}\left(\left(\kappa / \kappa_{c}\right) \pi d\right)\right] \mathrm{d} \kappa
$$

Computationally efficient asymptotic approximations for $Q\left(\kappa_{c}, d\right)$ as $\kappa_{c} \rightarrow 0$, similar to Voelk1 et al. (2000), are presently used.

We now choose the averaging operator to be the ensemble average,

$$
\langle\phi\rangle=\frac{1}{N} \sum_{x^{\prime} \in \mathscr{N}(x)} \phi,
$$

where $\phi$ is either $F_{2}$ or $Q ; \mathscr{N}(\boldsymbol{x})$ is the set of all points near $\boldsymbol{x}$; and $N$ is the number of points in $\mathscr{N}(\boldsymbol{x})$. Presently $\mathscr{N}(\boldsymbol{x})$ consists of points within the closed 
$\left(2 \Delta_{x}\right) \times\left(2 \Delta_{y}\right) \times\left(2 \Delta_{z}\right)$ block centred on $\boldsymbol{x}$, that is

$$
\mathscr{N}(\boldsymbol{x})=\left\{\boldsymbol{x}^{\prime}: \boldsymbol{x}^{\prime} \neq \boldsymbol{x},\left|x-x^{\prime}\right| \leqslant \Delta_{x},\left|y-y^{\prime}\right| \leqslant \Delta_{y},\left|z-z^{\prime}\right| \leqslant \Delta_{z}\right\} ;
$$

correspondingly, $N=3^{3}-1=26$.

\subsection{Extended stretched-vortex SGS model}

The original stretched-vortex SGS model consists of an ensemble of local twodimensional two-component flows. That is only planar motions of planar velocities of these local flows are considered when calculating ensemble statistics. The stretchedvortex SGS model used by Misra \& Pullin (1997) and Voelkl et al. (2000) is largely based on this construction. Pullin \& Lundgren (2001) found that planar motions (no axial coordinate dependence) inside cylindrical vortices convecting both passive scalars and the axial velocity could also be analysed within the same stretched-spiral vortex framework. An asymptotic two-dimensional three-component flow solution exists for which the evolution of the axial velocity and of a passive scalar follows essentially the same mapped evolution equations (Pullin \& Lundgren 2001; O'Gorman \& Pullin 2003). Using a simplified version of analysis, Pullin (2000) developed a vortex-based, SGS scalar flux model (see also Hill et al. 2006).

These ideas motivate the present extension to the stretched-vortex SGS stress model (Misra \& Pullin 1997) by incorporating the added effects of axial velocity transport modelled as a passive scalar. Recapitulating the result for subgrid scalar flux (Pullin 2000),

$$
\widetilde{v_{i}^{\prime} \widetilde{c}}=-K_{s} \frac{\partial \widetilde{c}}{\partial y_{i}} \quad \text { if } \quad i=1,2, \quad \widetilde{v_{3}^{\prime} \widetilde{c}}=0,
$$

where $K_{s}=\gamma \Delta_{c} K^{1 / 2} / 2$. Here, $v_{i}$ is the vortex-frame velocity and $y_{i}$ is the vortex-frame spatial coordinate ( $y_{3}$ coincides with the vortex axis); $\widetilde{c}$ is the resolved passive scalar; $v_{i}^{\prime}$ is the vortex-frame subgrid velocity; and $\gamma$ is an $O(1)$ universal dimensionless constant, estimated by Pullin (2000) to be equal to unity. Equation (2.15) says that the turbulent fluctuations of a subgrid spiral vortex of size $\Delta_{c}$ and energy $K$ transports the passive scalar in a way that results in a net flux of resolved passive scalar against its gradient.

Assuming analogous axial velocity transport dynamics $\left(\widetilde{c}=\widetilde{v}_{3}\right)$, we write the analogue of (2.15),

$$
\widetilde{v_{i}^{\prime} \widetilde{v}_{3}}=-K_{s} \frac{\partial \widetilde{v}_{3}}{\partial y_{i}} \quad \text { if } \quad i=1,2, \quad \widetilde{v_{3}^{\prime} \widetilde{v}_{3}}=0 .
$$

We shall later allow for a different value of $\gamma$ appropriate to axial velocity transport (see $\S 3.5$ ). Rotating back to the laboratory frame using the identities

$$
\begin{aligned}
\delta_{i 3} \widetilde{v}_{3} & =\delta_{i 3}\left(\delta_{j 3} \widetilde{v}_{j}\right)=e_{i}^{v}\left(e_{j}^{v} \widetilde{u}_{j}\right), \\
\left(\delta_{i 1} \delta_{j 1}+\delta_{i 2} \delta_{j 2}\right) \partial / \partial y_{j} & =\left(\delta_{i j}-\delta_{i 3} \delta_{j 3}\right) \partial / \partial y_{j}=\left(\delta_{i j}-e_{i}^{v} e_{j}^{v}\right) \partial / \partial x_{j},
\end{aligned}
$$

we arrive at the tensor form

$$
\widetilde{u_{i}^{\prime} \widetilde{u}_{j}}=-K_{s}\left[e_{j}^{v} e_{k}^{v} \frac{\partial \widetilde{u}_{k}}{\partial x_{l}}\left(\delta_{l i}-e_{l}^{v} e_{i}^{v}\right)\right] .
$$

We add this, and its transpose (since the subgrid stress tensor is symmetric), to the existing terms, (2.4), to obtain the extended subgrid stress model: 


$$
\begin{aligned}
T_{i j} & =\widetilde{u_{i}^{\prime} u_{j}^{\prime}}+\widetilde{u_{i}^{\prime} \widetilde{u}_{j}}+\widetilde{\tilde{u}_{i} u_{j}^{\prime}} \\
& =K\left(\delta_{i j}-e_{i}^{v} e_{j}^{v}\right)-K_{s}\left[e_{j}^{v} e_{k}^{v} \frac{\partial \widetilde{u}_{k}}{\partial x_{l}}\left(\delta_{l i}-e_{l}^{v} e_{i}^{v}\right)+e_{i}^{v} e_{k}^{v} \frac{\partial \widetilde{u}_{k}}{\partial x_{l}}\left(\delta_{l j}-e_{l}^{v} e_{j}^{v}\right)\right] .
\end{aligned}
$$

Note that the form is invariant under axis rotations and to sign reversals of $\boldsymbol{e}^{v}$. In other words, the subgrid stresses are understood to be produced by pairs of counterrotating SGS vortices. Also, the new terms are traceless so that the SGS kinetic energy remains unchanged $\left(T_{i i}=2 \mathrm{~K}\right)$.

We will later use (2.19) in the development of a special SGS wall model while retaining the standard stretched-vortex model, with SGS vortex aligned with the most extensive eigenvector of the resolved strain-rate tensor, that is $\boldsymbol{e}^{v}=\boldsymbol{e}_{\widetilde{S}}$, within the main body of the flow.

\section{Near-wall SGS model: boundary treatment}

We now describe in detail the development of our wall-adjacent SGS model. This recognizes explicitly the highly anisotropic character of near-wall turbulence. The main idea is to integrate across the near-wall layer in a way that models the appropriate physics while providing a slip boundary condition at a raised virtual wall for the resolved-scale LES.

\subsection{Near-wall filtering}

A novel feature of our boundary treatment is an ODE governing the local wall shear stress that, when coupled with the LES, eliminates the need to resolve the near-wall region. In this section, we shall, without loss of generality, fix the location of the wall at $z=0$. We first define an $x y$-plane filter:

$$
\widetilde{\phi}(x, y, z, t)=\iint \phi\left(x^{\prime}, y^{\prime}, z, t\right) G\left(x-x^{\prime} ; \Delta_{f}\right) G\left(y-y^{\prime} ; \Delta_{f}\right) \mathrm{d} x^{\prime} \mathrm{d} y^{\prime} .
$$

We require the filter width $\Delta_{f}$ to be much larger than the viscous wall unit $l^{+}$, where $l^{+} \equiv v / u_{\tau}$. We apply this filter to the streamwise momentum equation to obtain

$$
\frac{\partial \widetilde{u}}{\partial t}+\frac{\partial \widetilde{u u}}{\partial x}+\frac{\partial \widetilde{u v}}{\partial y}+\frac{\partial \widetilde{u w}}{\partial z}=-\frac{\partial \widetilde{p}}{\partial x}+v \frac{\partial^{2} \widetilde{u}}{\partial z^{2}},
$$

where we have neglected lateral diffusion, which is justifiably small, given the relative size of the filter width. The planar filtering is purely formal; we do not perform such filtering or indeed any explicit filtering in the present approach. In terms of inner variables, $\Delta_{f} \gg l^{+}$, where $l^{+}=\left(v / \eta_{0}\right)^{1 / 2}$ is the viscous, near-wall length scale and the wall velocity gradient $\eta_{0}$ is defined below. In outer variables $\Delta_{f} \ll \delta$, and we therefore consider $G$ in (3.1) as a delta function for the purposes of LES. The nonlinear terms include both resolved and subgrid contributions, $\widetilde{u_{i} u_{j}}=\widetilde{u}_{i} \widetilde{u}_{j}+T_{i j}$, and, for notational convenience, we have bundled the fluctuating pressure gradient with the mean pressure gradient, renaming $-\partial \widetilde{p} / \partial x+f(t)$ as $-\partial \widetilde{p} / \partial x$.

We next define a second, top-hat filter, which is an average in a wall-adjacent layer of arbitrary thickness $h$,

$$
\langle\phi\rangle(x, y, t)=\frac{1}{h} \int_{0}^{h} \widetilde{\phi}(x, y, z, t) \mathrm{d} z .
$$

Applying this to (3.2) along with the no-slip condition, $\widetilde{u}(x, y, 0, t)=0$, and assuming that the streamwise pressure gradient is constant throughout this wall-adjacent layer, 
we obtain

$$
\frac{\partial\langle u\rangle}{\partial t}+\frac{\partial\langle u u\rangle}{\partial x}+\frac{\partial\langle u v\rangle}{\partial y}=-\left.\frac{1}{h} \widetilde{u w}\right|_{h}-\left.\frac{\partial \widetilde{p}}{\partial x}\right|_{h}+\frac{v}{h}\left(\left.\frac{\partial \widetilde{u}}{\partial z}\right|_{h}-\eta_{0}\right),
$$

where the local wall streamwise velocity gradient is

$$
\left.\eta_{0} \equiv \frac{\partial \widetilde{u}}{\partial z}\right|_{0}
$$

The local wall shear stress is $\tau_{0}(x, y, t)=v \eta_{0}(x, y, t) \equiv u_{\tau}^{2}(x, y, t)$, where $u_{\tau}(x, y, t)$ is the local wall-friction velocity. Since $\Delta_{f} \gg l^{+}$, each computational cell is effectively assumed to behave as a local statistically homogeneous unit of wall turbulence, similar to the so-called minimal flow unit of Jiménez \& Moin (1991). Experiments conducted by Nakayama, Noda \& Maeda (2004) suggest $\Delta_{f}>1800 l^{+}$.

\subsection{Local inner scaling}

We now introduce a local inner-scaling ansatz. This states that the statistics of each cell are characterized by their respective local inner scales $v$ and $\eta_{0}(x, y, t)$ or equivalently $l^{+}$and $u_{\tau}(x, y, t)$. Specifically, we assert that the SGS streamwise velocity, within a near-wall, sugbrid region to be defined subsequently, can, for each cell, be collapsed onto the form

$$
\widetilde{u}(x, y, z, t)=\left(v \eta_{0}(x, y, t)\right)^{1 / 2} F\left(z^{+}\right), \quad z^{+}=z\left(\eta_{0}(x, y, t) / v\right)^{1 / 2} \equiv z / l^{+},
$$

where $F\left(z^{+}\right)$can be thought of as a local 'law of the wall'. Next, differentiate (3.6) with respect to $\eta_{0}$ to give

$$
\frac{\partial \widetilde{u}}{\partial \eta_{0}}=\frac{1}{2}\left(v / \eta_{0}\right)^{1 / 2}\left[F+z^{+} F^{\prime}\right]
$$

where $F^{\prime}=\mathrm{d} F / \mathrm{d} z^{+}$; perform the wall-adjacent average (3.3); and then back-substitute the inner scaling (3.6), evaluated at $z=h$,

$$
\frac{\partial\langle u\rangle}{\partial \eta_{0}}=\frac{1}{2}\left(v / \eta_{0}\right)^{1 / 2} F\left(h^{+}\right)=\frac{\left.\widetilde{u}\right|_{h}}{2 \eta_{0}},
$$

where $\left.\widetilde{u}\right|_{h}=F\left(h^{+}\right)$with $h^{+}=h / l^{+}$. Finally, using the chain rule we find

$$
\frac{\partial\langle u\rangle}{\partial t}=\frac{\partial\langle u\rangle}{\partial \eta_{0}} \frac{\partial \eta_{0}}{\partial t}=\frac{\left.\widetilde{u}\right|_{h}}{2 \eta_{0}} \frac{\partial \eta_{0}}{\partial t} .
$$

We emphasize that (3.9) is an exact consequence of (3.3) and (3.6). Moreover, using (3.9) to evaluate the time derivative of the plane-filtered and vertically averaged streamwise velocity, the explicit form of $F\left(z^{+}\right)$in $0 \leqslant z<h$ is not needed; this occurs owing to the cancellation of two integrals. The velocity $\left.\widetilde{u}\right|_{h}$ will later be obtained directly from the LES.

Now substitute (3.9) into the first term of (3.4) to obtain

$$
\frac{\left.\widetilde{u}\right|_{h}}{2 \eta_{0}} \frac{\partial \eta_{0}}{\partial t}+\frac{\partial\langle u u\rangle}{\partial x}+\frac{\partial\langle u v\rangle}{\partial y}=-\left.\frac{1}{h} \widetilde{u w}\right|_{h}-\left.\frac{\partial \widetilde{p}}{\partial x}\right|_{h}+\frac{v}{h}\left(\left.\frac{\partial \widetilde{u}}{\partial z}\right|_{h}-\eta_{0}\right) .
$$

Our motivation for performing the wall-adjacent average, (3.3), is to remove the steep near-wall gradients, which we do not wish to resolve. In-plane $(x, y)$ gradients of filtered quantities in (3.10) are now approximated by values at $z=h$, supplied by the 


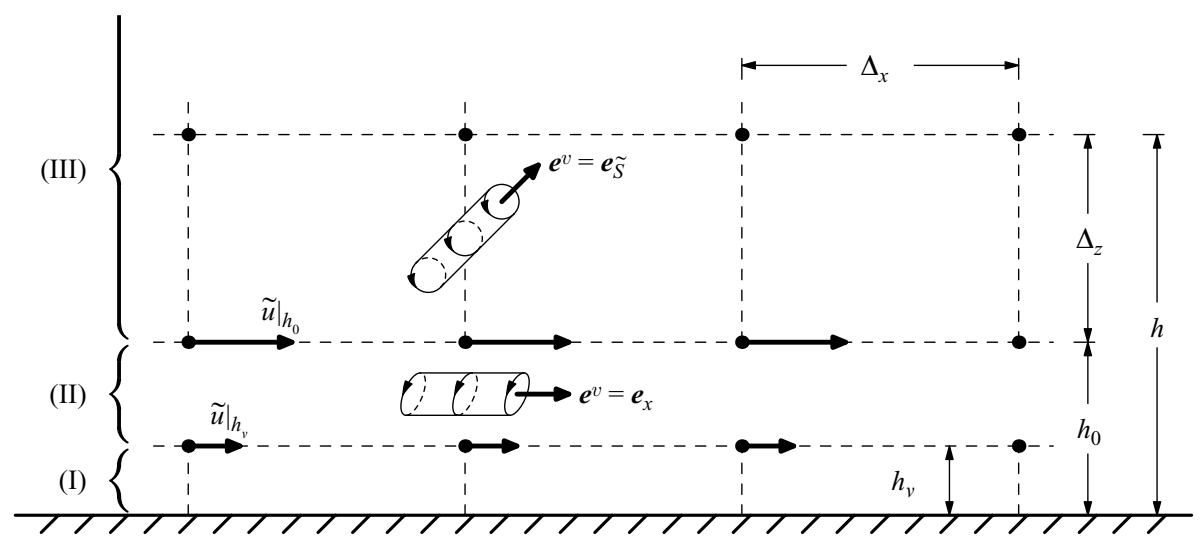

FIGURE 1. Schematic showing the near-wall set-up: $h_{0}$ locates the lifted virtual wall, where boundary conditions are applied; $h$ locates the input plane to the wall shear stress equation, $(3.10) ; h_{v}$ locates the outer edge of the viscous sublayer; $\boldsymbol{e}^{v}$ is the alignment of SGS vortices in their respective regions.

LES,

$$
\frac{\partial\langle u u\rangle}{\partial x} \approx \frac{\left.\partial \widetilde{u u}\right|_{h}}{\partial x}, \quad \frac{\partial\langle u v\rangle}{\partial y} \approx \frac{\left.\partial \widetilde{u v}\right|_{h}}{\partial y} .
$$

This approximation captures some of the non-equilibrium effects arising from largescale in-plane inhomogeneities. With these assumptions, we rewrite (3.10) as

$$
\frac{\partial \eta_{0}}{\partial t}=\frac{2 \eta_{0}}{\left.\widetilde{u}\right|_{h}}\left[-\left.\frac{1}{h} \widetilde{u w}\right|_{h}-\frac{\left.\partial \widetilde{u u}\right|_{h}}{\partial x}-\frac{\left.\partial \widetilde{u v}\right|_{h}}{\partial y}-\left.\frac{\partial \widetilde{p}}{\partial x}\right|_{h}+\frac{v}{h}\left(\left.\frac{\partial \widetilde{u}}{\partial z}\right|_{h}-\eta_{0}\right)\right] .
$$

Equation (3.12) governs the evolution of the wall shear stress, written in terms of $\eta_{0}$. The right-hand side of (3.12) only involves known quantities at $h$, which is arbitrary. In practice, we choose $h$ to be at the first grid point within the LES (see figure 1), and the quantities on the right-hand side can then be determined from resolved-scale LES quantities. We remark that our use of local inner scaling, (3.6), is restricted to the reduction of the unsteady term in (3.4) and that this operation does not require a specific form for $F\left(z^{+}\right)$. The other terms in (3.12) will be provided from the resolved-scale LES itself, so that (3.12) can then be viewed in this sense as resulting from mixed inner-outer scaling. When coupled with an LES, (3.12) then allows us to determine the wall shear stress without resolving the near-wall steep gradients, which have been integrated out by the wall averaging. One can also interpret (3.12) as an integrated form of the local unsteady turbulent boundary layer equations with the added assumption of local inner scaling for the unsteady term. Further, (3.12) knows nothing about the channel geometry and should, therefore, be applicable to general flows. To close this coupling, appropriate boundary conditions for the LES need to be applied, which is the subject of the next subsection.

\subsection{Multi-layer SGS wall model}

We do not resolve the near-wall region. Instead, the LES computation takes place above a certain fixed, $R e$-independent height $h_{0}$, which will later be chosen as a small fraction of the near-wall cell size. To proceed we first define three regions for the lower half-channel. It is understood that, for the present simulations, similar regions 
exist on the upper wall. These regions are (see figure 1)

(i) $0 \leqslant z \leqslant h_{v}$, region (I), essentially the viscous sublayer;

(ii) $h_{v}<z \leqslant h_{0}$, region (II), viewed as an overlap layer, where the shear stress is approximately constant and will be modelled by the extended stretched-vortex SGS model consisting of attached vortices aligned with $\boldsymbol{e}_{x}$; and

(iii) $h_{0}<z \leqslant \delta$, region (III), where non-universal outer flow features are computed with LES coupled with the original stretched-vortex SGS model of detached subgrid vortices aligned with $\boldsymbol{e}_{\tilde{S}}$.

We remark that the combination of attached and detached vortices was also used by Marušic $\&$ Perry (1995) to model wall turbulence. The plane $z=h$ lies at the top of the first grid cell in region (III). The plane $z=h_{0}$ will be referred to as the lifted virtual wall. We now proceed to model the flow in regions (I) and (II) in a way that provides a slip velocity at $z=h_{0}$.

In region (I) we use $\widetilde{u}^{+}=z^{+}$, where $\widetilde{u}^{+}=\widetilde{u} / u_{\tau}, z^{+}=z / l^{+}$, and $u_{\tau}$ is known. In particular, $\left.\widetilde{u}^{+}\right|_{h_{v}}=h_{v}^{+}$, where $h_{v}^{+}=h_{v} / l^{+}$. For a hydrodynamically smooth wall, where the wall roughness is small compared to $l^{+}$, experiments indicate that the outer edge of the viscous sublayer is located at $h_{v}^{+} \approx 11$ (based on the intercept between the linear and $\log$ components of the law of the wall). We will therefore take $\left.\widetilde{u}^{+}\right|_{h_{v}}=h_{v}^{+}=11$. In fact, this intercept is found to be sensitive to pressure gradients and can assume values in the range 10-15 (Nickels 2004). A cubic equation was successfully used in the paper of Nickels (2004) to model this effect; we do not pursue this presently in favour of simplicity, although this generalization should certainly be included for separating flows. Above $z^{+}=h_{v}^{+}$, inviscid outer flow dynamics become important.

\subsection{Slip velocity at lifted virtual wall}

We now model the mean-flow dynamics in region (II), $h_{v}<z \leqslant h_{0}$. We require $h_{0}$ to scale with outer flow thickness $\delta$ but to remain relatively small, $h_{0}<0.1 \delta$, say, so that non-universal effects (the wake) uncharacteristic of the inner scales can be captured by the LES in region (III). This will permit the LES to be performed with the same grid for a wide range of $R e$, eliminating the $O\left(R e_{\tau}^{1.8}\right)$ scaling requirement for the grid resolution of a partially resolved wall-bounded LES (Piomelli 2008). Put another way, $h_{0}$ remains fixed, $O(\delta)$, but $h_{v}$ becomes thinner, $O\left(l^{+}\right)$, with increasing $R e$.

Region (II) is, by construction, the so-called overlap region, or the productionequals-dissipation layer, where the shear stress is approximately constant. Furthermore, the shear stress is balanced by the wall shear stress (Townsend 1976). Casting these ideas in LES terminology,

$$
u_{\tau}^{2}(x, y, t)=-\widetilde{u w}=-\widetilde{u} \widetilde{w}-T_{x z}=-T_{x z}, \quad \text { since } \widetilde{w}=0 .
$$

The existence of quasi-streamwise vortical structures in wall turbulence have long been observed by researchers (see e.g. Head \& Bandyopadhyay 1981; Robinson 1991) and have also served as useful physical models (see e.g. Townsend 1976; Perry \& Chong 1982; Marušić \& Perry 1995; Adrian 2007; Nickels et al. 2007). Motivated by these studies, we model region (II) with an ensemble of vortices aligned in the streamwise direction, $\left(e_{x}^{v}, e_{y}^{v}, e_{z}^{v}\right)=(1,0,0) \Leftrightarrow \boldsymbol{e}^{v}=\boldsymbol{e}_{x}$. Substituting these into the expression for the shear stress produced by the extended stretched-spiral vortex SGS model, (2.19), and noting that the only non-zero component of the mean velocity gradient tensor is $\mathrm{d} \widetilde{u} / \mathrm{d} z$, we obtain

$$
T_{x z}=-\frac{1}{2} \gamma_{\text {II }} K^{1 / 2} \Delta_{c} \frac{\mathrm{d} \widetilde{u}}{\mathrm{~d} z} .
$$




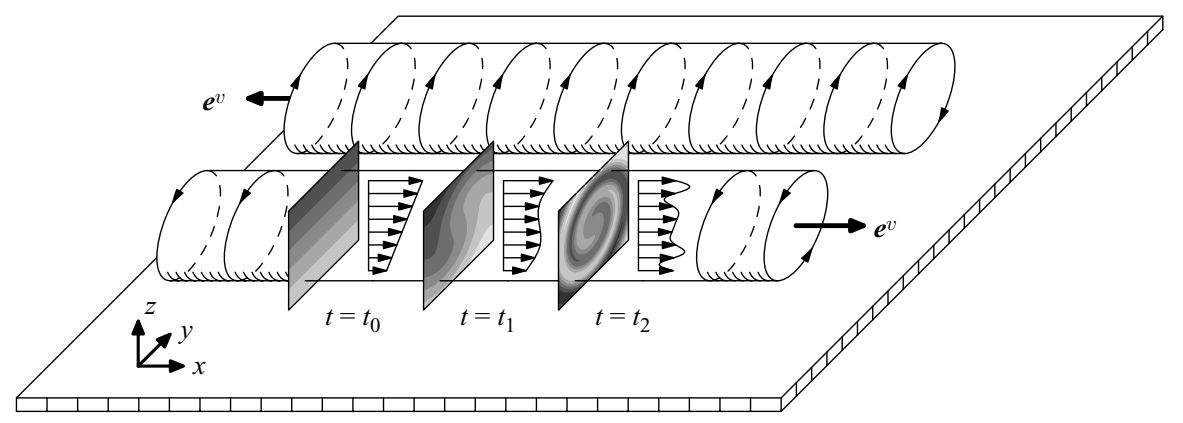

FIGURE 2. Schematic of a pair of attached counter-rotating vortices. The winding effect of the vortices are shown on contour plots of the streamwise velocity along with the accompanying profiles. Darker shades represent higher-momentum fluid. The various stages of mixing are characterized by the times $t=t_{0}<t_{1}<t_{2}$.

Recall the physical mechanism that produces this shear stress: the action of the spiralling streamwise vortex is to wrap its own axial velocity, now identified as the mean streamwise velocity, as if it were a passive scalar (see figure 2), thereby transporting high-momentum fluid towards the wall and transporting low-momentum fluid away from the wall. This process has the observed effect of a flattened streamwise velocity profile.

Unlike the SGS vortices in region (III), which are unaware of the presence of the wall and are, therefore, considered as detached from the wall, the size of these near-wall vortices, $\Delta_{c}$, are constrained by the presence of the wall so that $\Delta_{c}=z$. That is (3.14) with $\Delta_{c} \rightarrow z$ can be interpreted as the shear stress produced by a hierarchy of longitudinal vortices that scale with the wall distance. This scaling assumption is, in fact, the idea of the so-called attached wall eddy (Nickels et al. 2007). We therefore write (3.14) in the form

$$
\frac{\mathrm{d} \widetilde{u}}{\mathrm{~d} z}=\frac{1}{\mathscr{K}_{1}} \frac{u_{\tau}}{z},
$$

where the dimensionless local quantity given by

$$
\mathscr{K}_{1}(x, y, t)=\frac{\gamma_{\mathrm{II}} K^{1 / 2}}{2\left(-T_{x z} / u_{\tau}\right)}
$$

resembles the Kármán constant $\kappa$. An implicit assumption in the derivation of (3.15) is that $K$ is sensibly independent of $z$, even though $\Delta_{c}$ decreases as the wall is approached. This is possible if the number of these vortices (population density) also increase in proportion to their decrease in size in order to maintain the same $K$.

Recall that $K$ is also the SGS kinetic energy of the vortices lying in region (II) and should be obtained from the structure-function-matching procedure local to the vortex location. However, since, by construction, no grid points are placed within region (II), we will use the grid points just inside the LES domain, centred on the plane, $z=h=h_{0}+\Delta_{z}$, for this purpose.

In region (II), we now consider $T_{x z}$ as constant in (3.16) and model this as the geometric average of its value at the true wall and at the top of region (II), so that

$$
-T_{x z}=u_{\tau}\left(-\left.T_{x z}\right|_{e_{\tilde{s}}}\right)^{1 / 2}
$$


Hence (3.16) becomes

$$
\mathscr{K}_{1}=\frac{\gamma_{\mathrm{II}} K^{1 / 2}}{2\left(-\left.T_{x z}\right|_{\boldsymbol{e}_{\tilde{S}}}\right)^{1 / 2}} .
$$

Equation (3.18), used in the limit $\kappa_{c} \rightarrow 0$ for increased robustness (to avoid division by small numbers), provides a way to calculate the local Kármán constant. Solving (3.15) in region (II), $h_{v}<z \leqslant h_{0}$, and evaluating the result at $z=h_{0}$ yields

$$
\left.\widetilde{u}\right|_{h_{0}}=\frac{u_{\tau}}{\mathscr{K}_{1}} \log \left(\frac{h_{0}}{h_{v}}\right)+\left.\widetilde{u}\right|_{h_{v}}=u_{\tau}\left(\frac{1}{\mathscr{K}_{1}} \log \left(\frac{h_{0}}{h_{v}}\right)+h_{v}^{+}\right),
$$

where the constant of integration has been chosen by putting $\left.\widetilde{u}\right|_{h_{v}}=u_{\tau} h_{v}^{+}$. We will use (3.19), which serves as a jump condition between the $h_{v}-h_{0}$ planes, along with $\widetilde{v}=0$ and $\widetilde{w}=0$, to set the Dirichlet boundary conditions at the lifted virtual wall $h_{0}$; $u_{\tau}$ is obtained from the solution of (3.12).

Equation (3.19) has been obtained from a physical model of region (II) in which the dominant Reynolds shear stress is modelled by streamwise-aligned vortices that transport low-momentum mean streamwise velocity away from the wall and highmomentum mean streamwise velocity towards the wall. The idea that these self-similar vortices scale only with $z$-independent of $l^{+}$and $\delta$-implies an overlap argument. Equation (3.19) couples $\left.\widetilde{u}\right|_{h_{0}}$ with the resolved-scale LES in region (III), $h_{0}<z \leqslant \delta$, which provides both $K$ and $\boldsymbol{e}_{\widetilde{\varsigma}}$. Equation (3.19) contains two constants, $\gamma$ and $h_{v}^{+}$. The latter is given empirically by $h_{v}^{+}=11$ from our discussion of region (I); a different value could be used for rough-wall flows. This physical model provides a means of dynamically calculating the instantaneous local 'Kármán constant' $\mathscr{K}_{1}$ as part of the LES. This will be demonstrated later.

\subsection{Estimation of the mixing time constant $\gamma_{\mathrm{II}}$}

A constant, $\gamma_{\text {II }}$, is required in (3.19). Owing to the highly anisotropic character of near-wall turbulent physics, this is expected to be somewhat different in value from that used in the SGS scalar application (Pullin 2000).

Consider the interface of regions (II) and (III), $z=h_{0}$, where both inner and outer layer modelling ideas are valid; in the spirit of LES filtering, we interpret this interface as a blurred boundary between the two regions so that the change in underlying vortical flow is gradual. This interface, $z \approx h_{0}<0.1 \delta$, is near the wall, so the LES filtered flow field can be approximated by simple shear flow, $\partial \widetilde{u}_{i} / \partial x_{j}=\delta_{i 1} \delta_{j 3} S$, where $S=\mathrm{d} \widetilde{u} / \mathrm{d} z$. This implies that, in this region, $\boldsymbol{e}_{\widetilde{S}}=(1 / \sqrt{2}, 0,1 / \sqrt{2})$; that is the detached vortices are inclined at $45^{\circ}$ to the wall.

We estimate $\gamma_{\text {II }}$ by matching Townsend's structure parameter,

$$
a_{1}=T_{13} / T_{i i}=T_{13} /(2 K),
$$

of the two vortical flow descriptions at this interface region. Given the vortex alignment, this parameter measures the amount of shear stress that can be supported relative to the vortex kinetic energy. First, the $\boldsymbol{e}_{\widetilde{S}}$ alignment and (2.19) give

$$
\left.a_{1}\right|_{\tilde{S}}=\left(-\left.K\right|_{e_{\tilde{S}}} / 2\right) /\left(\left.2 K\right|_{e_{\tilde{S}}}\right)=-1 / 4 .
$$

Similarly, the $\boldsymbol{e}_{x}$ alignment and (2.19) give

$$
\left.a_{1}\right|_{e_{x}}=\left(-\gamma_{\mathrm{II}}\left(\left.K\right|_{\boldsymbol{e}_{x}}\right)^{1 / 2} \Delta_{c} S / 2\right) /\left(\left.2 K\right|_{\boldsymbol{e}_{x}}\right)=-\gamma_{\mathrm{II}} \Delta_{c} S\left(\left.K\right|_{\boldsymbol{e}_{x}}\right)^{-1 / 2} / 4 .
$$


To proceed, we assume high $R e$ so that $\kappa_{c} \rightarrow 0$. Also, for simplicity, $d \rightarrow 0$. The subgrid kinetic energy of the streamwise vortex then reduces to

$$
\left.K\right|_{e_{x}}=2\left\langle\left(\delta u_{i}\right)^{2}\right\rangle /\left(\pi^{2}\left\langle d^{2}\right\rangle\right)
$$

The local averaging is dominated by the background shear, so we can approximate $\left\langle\left(\delta \widetilde{u}_{i}\right)^{2}\right\rangle \approx\left\langle(\delta \widetilde{u})^{2}\right\rangle \approx\left(\Delta_{z} S\right)^{2}$, and for the same reason $\left\langle d^{2}\right\rangle=\left(\Delta_{z} / \Delta_{c}\right)^{2}$. Then

$$
\left.K\right|_{e_{x}}=2\left(\Delta_{c} S / \pi\right)^{2} \text {. }
$$

Using this, we obtain $a_{1}$ for the $\boldsymbol{e}_{x}$ aligned vortex,

$$
\left.a_{1}\right|_{\boldsymbol{e}_{x}}=-2^{-5 / 2} \pi \gamma_{\mathrm{II}}
$$

Finally, matching these, $\left.a_{1}\right|_{e_{\widetilde{S}}}=\left.a_{1}\right|_{e_{x}}$, we have $\gamma_{I I}=2^{1 / 2} / \pi \approx 0.45$. This is the value used presently for all LES.

\subsection{Summary of SGS wall model}

Our SGS model for the near-wall dynamics can be summarized as follows: for every cell adjacent to both the top and bottom walls, (3.12) is solved for $\eta_{0}$ with terms on the right-hand side provided by the LES at the top of the wall-adjacent cell at $z=h=\Delta_{z}+h_{0}$. This provides $\eta_{0}(x, y, t)$ and thus $u_{\tau}(x, y, t)$. Equation (3.19) is then used to evaluate the streamwise slip velocity $\left.\widetilde{u}\right|_{h_{0}}(x, y, t)$ at $z=h_{0}$, with $\mathscr{K}_{1}$ evaluated from (3.18) and with $K$ and $\left.T_{x z}\right|_{\boldsymbol{e}_{\tilde{S}}}$ evaluated at $z=h=\Delta_{z}+h_{0}$ from the LES structure-function-matching procedure. The other boundary conditions at the virtual wall are taken as $\left.\widetilde{v}\right|_{h_{0}}(x, y, t)=\left.\widetilde{w}\right|_{h_{0}}(x, y, t)=0$. This method couples the LES with the modelled, near-wall dynamics. The LES has implicit knowledge of the true no-slip boundary condition, because this was used in obtaining (3.12), and the smooth-wall condition through use of $h_{v}^{+}=11$. Because the LES quantities in both (3.12) and (3.18) are evaluated at the top of the first cell at $z=h=\Delta_{z}+h_{0}$, the height of the virtual wall at $z=h_{0}$ should satisfy $h_{v}<h_{0}<h$. Presently we use $h_{0}=0.18 \Delta_{z}$, independent of the LES resolution, and consider this as part of the overall grid. Some tests to investigate sensitivity to $h_{0}$ were performed.

The governing equations are advanced using a third-order semi-implicit RungeKutta method coupled with the Kleiser-Schumann influence-matrix method for enforcing the divergence-free constraint. The spatial discretization employs the Fourier spectral method for the wall-parallel planes and a fourth-order accurate finite difference on a uniformly spaced grid for the wall-normal direction. These are detailed in Appendix A.

\section{Results and discussion}

The LES presently performed are summarized in table 1. Four sets were done and are labelled A, B, C and D. All used the same form of the wall SGS model with $\gamma_{\mathrm{II}}=0.45$. For sets $\mathrm{A}$ and $\mathrm{B}$, the original form of the stretched-vortex SGS model was used in region (III). This corresponds to $\gamma_{\mathrm{III}}=0$ in (2.19). For set $\mathrm{C}, \gamma_{\mathrm{III}}=0.45$ was used in (2.19). There is no inconsistency with $\gamma_{\mathrm{II}} \neq \gamma_{\mathrm{III}}$ : the former is a necessary part of the special SGS model in region (II), while the latter forms part of the global SGS model for LES in region (III). Set F is used to evaluate the wall model's sensitivity to the virtual wall location $h_{0}$. The simulations are run until the first- and second-order statistics have converged. Data is then collected from one snapshot in time; our interest is in low-order statistics, which do not benefit significantly from time averaging. 


\begin{tabular}{lcrrrrrrrrrrr}
\hline Case & $R e_{\mathrm{c}}$ & $R e_{\tau}$ & $\eta(\%)$ & $L_{x} / \delta^{\prime}$ & $L_{y} / \delta^{\prime}$ & $h_{0} / \Delta_{z}$ & $N_{x}$ & $N_{y}$ & $N_{z}$ & $\Delta_{x}^{+}$ & $\gamma_{\mathrm{III}}$ & $\overline{\mathscr{K}}_{1}$ \\
$\mathrm{~A} 1$ & $49 \mathrm{k}$ & $2 \mathrm{k}$ & 1.1 & 32 & 8 & 0.18 & 192 & 48 & 48 & $3.3 \times 10^{2}$ & 0 & 0.37 \\
$\mathrm{~A} 2$ & $610 \mathrm{k}$ & $20 \mathrm{k}$ & 2.2 & 32 & 8 & 0.18 & 192 & 48 & 48 & $3.3 \times 10^{3}$ & 0 & 0.38 \\
$\mathrm{~A} 3$ & $7400 \mathrm{k}$ & $200 \mathrm{k}$ & 2.9 & 32 & 8 & 0.18 & 192 & 48 & 48 & $3.3 \times 10^{4}$ & 0 & 0.38 \\
$\mathrm{~A} 4$ & $87 \mathrm{M}$ & $2 \mathrm{M}$ & 2.1 & 32 & 8 & 0.18 & 192 & 48 & 48 & $3.3 \times 10^{5}$ & 0 & 0.37 \\
$\mathrm{~A} 5$ & $990 \mathrm{M}$ & $20 \mathrm{M}$ & 1.7 & 32 & 8 & 0.18 & 192 & 48 & 48 & $3.3 \times 10^{6}$ & 0 & 0.37 \\
$\mathrm{~B} 2$ & $610 \mathrm{k}$ & $20 \mathrm{k}$ & 2.9 & 32 & 8 & 0.18 & 384 & 96 & 96 & $1.7 \times 10^{3}$ & 0 & 0.38 \\
$\mathrm{~B} 3$ & $7400 \mathrm{k}$ & $200 \mathrm{k}$ & 2.7 & 32 & 8 & 0.18 & 384 & 96 & 96 & $1.7 \times 10^{4}$ & 0 & 0.38 \\
$\mathrm{~B} 4$ & $86 \mathrm{M}$ & $2 \mathrm{M}$ & 2.2 & 32 & 8 & 0.18 & 384 & 96 & 96 & $1.7 \times 10^{5}$ & 0 & 0.38 \\
$\mathrm{C} 2$ & $580 \mathrm{k}$ & $20 \mathrm{k}$ & 3.6 & 32 & 8 & 0.18 & 192 & 48 & 48 & $3.3 \times 10^{3}$ & 0.45 & 0.39 \\
$\mathrm{C} 3$ & $6900 \mathrm{k}$ & $200 \mathrm{k}$ & 1.0 & 32 & 8 & 0.18 & 192 & 48 & 48 & $3.3 \times 10^{4}$ & 0.45 & 0.40 \\
$\mathrm{C} 4$ & $81 \mathrm{M}$ & $2 \mathrm{M}$ & -0.4 & 32 & 8 & 0.18 & 192 & 48 & 48 & $3.3 \times 10^{5}$ & 0.45 & 0.39 \\
$\mathrm{~F} 2$ & $620 \mathrm{k}$ & $20 \mathrm{k}$ & -0.8 & 32 & 8 & 0.36 & 192 & 48 & 48 & $3.3 \times 10^{3}$ & 0 & 0.38 \\
$\mathrm{~F} 3$ & $7600 \mathrm{k}$ & $200 \mathrm{k}$ & 0.6 & 32 & 8 & 0.36 & 192 & 48 & 48 & $3.3 \times 10^{4}$ & 0 & 0.38 \\
$\mathrm{~F} 4$ & $89 \mathrm{M}$ & $2 \mathrm{M}$ & 0.8 & 32 & 8 & 0.36 & 192 & 48 & 48 & $3.3 \times 10^{5}$ & 0 & 0.38
\end{tabular}

TABLE 1. Simulation parameters and outputs: $\mathrm{k} \equiv 10^{3}, \mathrm{M} \equiv 10^{6} ; R e_{\mathrm{c}} \equiv u_{\mathrm{c}} \delta / v ; u_{\mathrm{c}}$ is the mean centreline velocity; $R e_{\tau} \equiv u_{\tau} \delta / v ; \eta \equiv\left(R e_{\tau}\right)_{a c t} / R e_{\tau}-1 ; \delta^{\prime} \equiv L_{z} / 2=\delta-h_{0} \approx \delta ; \Delta_{x}=\Delta_{y}=4 \Delta_{z}$; $R e_{\tau}$ is nominal; $\left(R e_{\tau}\right)_{a c t}$ is actual; $\gamma_{\text {III }}$ refers to $\gamma$ in (2.19) used in the interior of the LES domain.

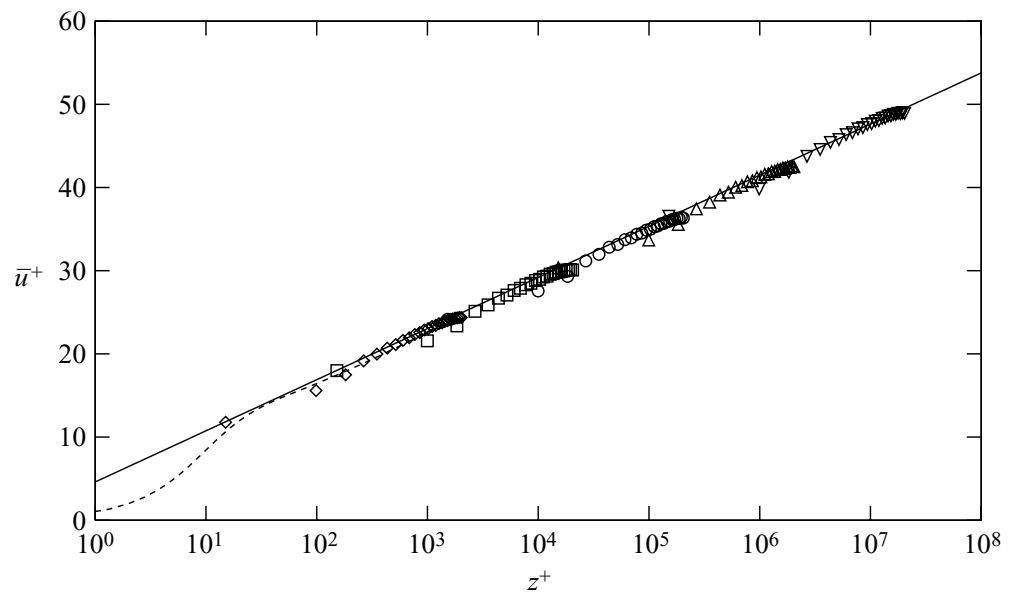

FIGURE 3. Mean velocity profiles for LES with $\gamma_{\mathrm{III}}=0$ (set A): $\diamond, R e_{\tau}=2 \mathrm{k} ; \square, R e_{\tau}=20 \mathrm{k}$; $\bigcirc$, $R e_{\tau}=200 \mathrm{k} ; \triangle, R e_{\tau}=2 \mathrm{M} ; \nabla, R e_{\tau}=20 \mathrm{M} ;----, R e_{\tau}=2 \mathrm{k}$ DNS (Hoyas \& Jiménez 2006); $\longrightarrow, \log \left(z^{+} / 11\right) / 0.37+11$.

\subsection{Profiles}

The mean streamwise velocity profiles for $R e_{\tau}=2 \mathrm{k}-20 \mathrm{M}$ (set A) corresponding to the original LES model $\left(\gamma_{\mathrm{III}}=0\right)$ coupled with the present wall model are shown in figure 3, where $\overline{()}$ denotes the $x y$-plane average at a particular time. Also shown is the $\log$ relationship (3.19) with the predicted $\overline{\mathscr{K}}_{1}$ averaged across all cases, $\left(\overline{\mathscr{K}}_{1}\right)_{\text {avg }}=0.37$. Its intercept is $11-\log (11) / 0.37=4.5$ (compare with the classic value of $\approx 5$ ). The slight drop in the mean-flow profile for the two points adjacent to the lifted virtual wall was thought to be a manifestation of a spurious sublayer due to the Dirichlet slip and no-transpiration boundary conditions (Cabot \& Moin 1999). This issue is 

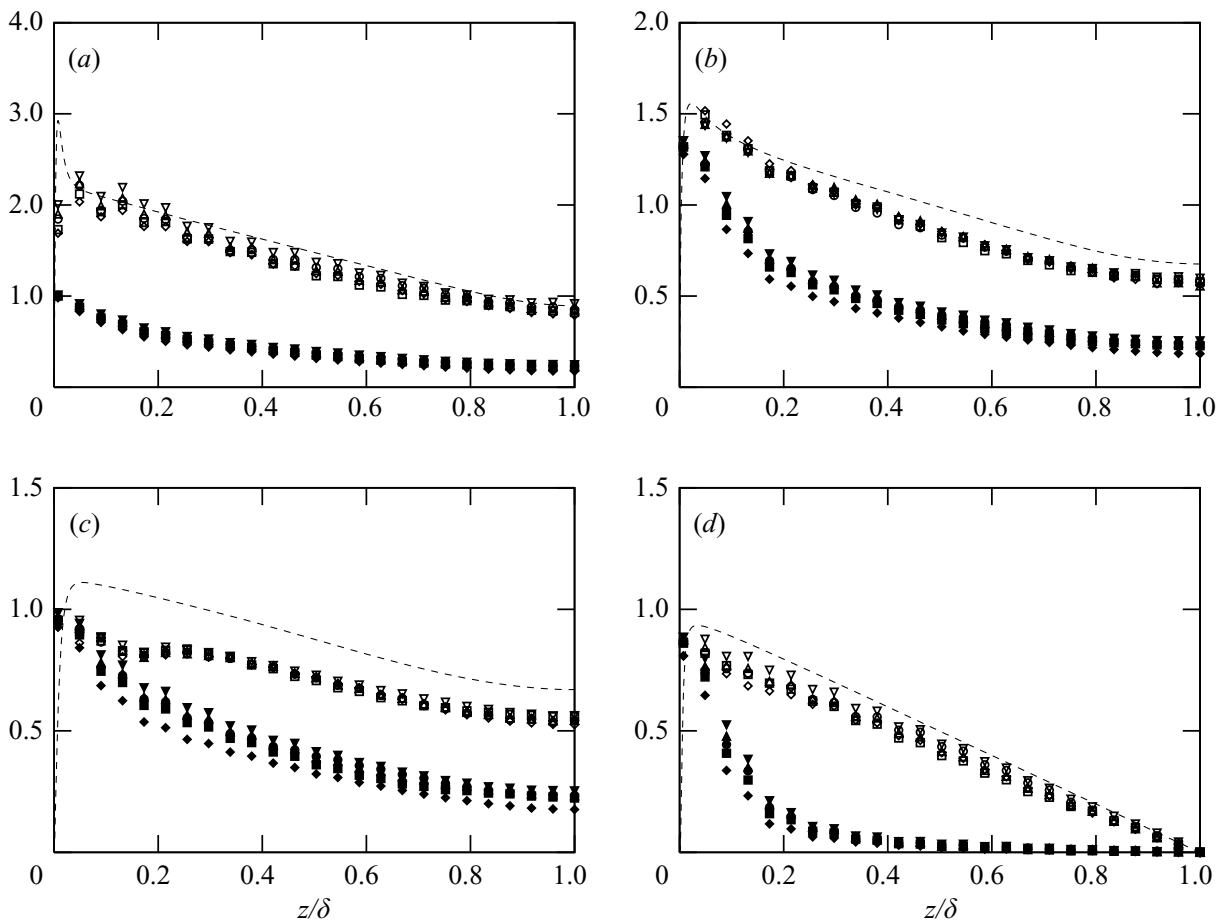

FiguRE 4. Turbulence statistics, (a) $u_{r m s}^{+}$, (b) $v_{r m s}^{+}$, (c) $w_{r m s}^{+}$and $(d)-\overline{u w}^{+}$, for LES with $\gamma_{\mathrm{III}}=0$ (set A) $\diamond, \operatorname{Re}_{\tau}=2 \mathrm{k} ; \square, \operatorname{Re}_{\tau}=20 \mathrm{k} ; O, \operatorname{Re}_{\tau}=200 \mathrm{k} ; \Delta, \operatorname{Re}_{\tau}=2 \mathrm{M} ; \nabla, \operatorname{Re}_{\tau}=20 \mathrm{M}$; ,$---- R e_{\tau}=2 \mathrm{k}$ DNS (Hoyas \& Jiménez 2006); open symbols, total (resolved plus subgrid); solid symbols, subgrid.

not specific to Dirichlet boundary conditions but can also be observed in wall stress boundary conditions (see Pantano et al. 2008). The flow quickly recovers beyond the two points and appears to follow the log profile.

We plot the corresponding profiles of the turbulent statistics in figure 4 , where ( ) rms refers to the root mean square (r.m.s.) of the fluctuations. The mild near-wall oscillations may suggest that we used an inadequate time-averaging interval. However, the localized nature of these oscillations and similar observations by Pantano et al. (2008) offer the explanation that they are, in fact, Gibbs oscillations caused by the singular no-penetration boundary condition.

Recently, the peak in near-wall $u$-fluctuations was observed to be mildly increasing with Reynolds number (DeGraaff \& Eaton 2000; Del Álamo et al. 2004). This peak, located at $z^{+} \approx 12$, is beneath our lifted wall, $z=h_{0}$, and well within our modelled near-wall layer consisting of streamwise vortices - region (II). Accordingly, we do not expect to capture this trend with statistics of the present simulations, which are essentially an outer-layer - region (III) - LES. This is a limitation of the present wall model.

The $w$-fluctuations are uniformly underpredicted; this is not surprising, since we used the no-transpiration boundary condition, which does not strictly hold at the lifted virtual wall. This amounts to letting the LES SGS model predict all near-wall $w$-fluctuations, as confirmed by the subgrid component in figure 4(c). The overall anisotropy in the Reynolds stresses, however, is preserved by our anisotropic LES model, even close to the wall. Physically, we are letting the LES model do the work 


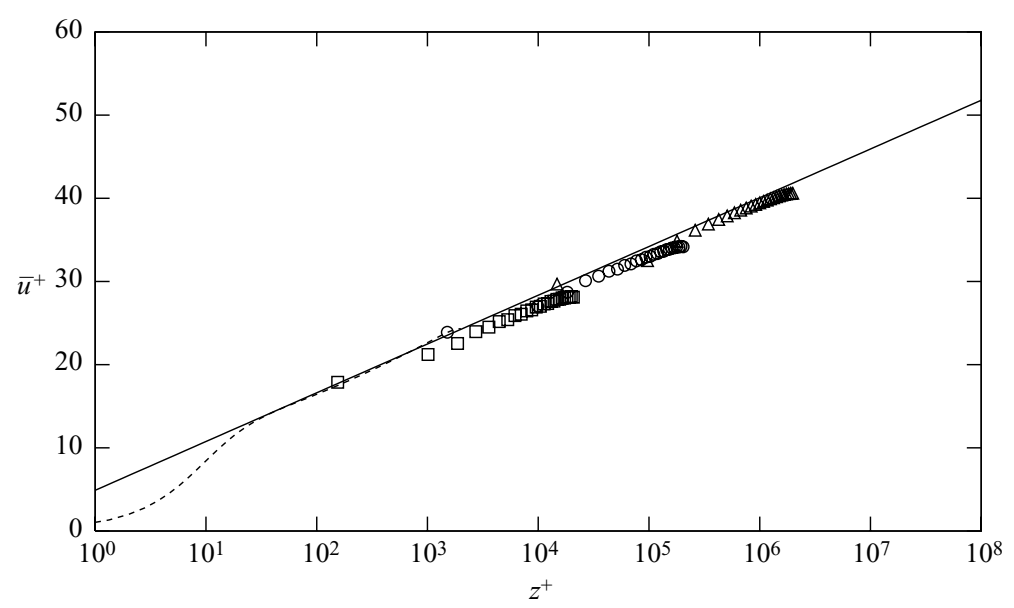

FigURE 5. Mean velocity profiles for LES with $\gamma_{\mathrm{III}}=0.45$ (set C): $\square, R e_{\tau}=20 \mathrm{k} ; \mathrm{O}$, $R e_{\tau}=200 \mathrm{k} ; \Delta, R e_{\tau}=2 \mathrm{M} ;----, R e_{\tau}=2 \mathrm{k}$ DNS (Hoyas \& Jiménez 2006); $\log \left(z^{+} / 11\right) / 0.39+11$.

of modelling the near-wall 'large eddies', since these, constrained by the wall, become part of the near-wall subgrid motion. This capability of the stretched-spiral vortex SGS model is also clearly shown by Pantano et al. (2008).

Near the wall $-\overline{u w}^{+}$is slightly smaller than unity (see figure $4 d$ ), indicating a small imbalance between $-\overline{u w}$, as determined by the LES SGS model, and $\overline{u_{\tau}^{2}}=\overline{v \eta_{0}}$, as determined by the ODE for $\eta_{0}$. To understand this, we set $\partial / \partial t=0$ in the ODE (3.12), solve for $\nu \eta_{0}$ and then take the plane average to reveal that this imbalance is caused by a non-vanishing plane average of $\left.\partial \widetilde{u u}\right|_{h} / \partial x$ and $\left.\partial \widetilde{u v}\right|_{h} / \partial y$, as these are numerically calculated in the skew-symmetric form. Similar trends in mean profiles and turbulence statistics are observed (not shown) when the resolution is doubled in all directions (set B).

The mean streamwise velocity profiles for $R e_{\tau}=20 \mathrm{k}-2 \mathrm{M}$ (set C) that are LES performed with the extended form for $T_{i j}$ given by (2.19) and $\gamma_{\text {III }}=0.45$ slightly underpredict the logarithmic relationship (see figure 5). The average of the predicted Kármán constant is 0.39 . The corresponding turbulent statistics are shown in figure 6. For these cases, the near-wall $w$-fluctuations are underpredicted (see figure $6 c$ ).

For LES with $\gamma_{\mathrm{III}}=0$ (sets A and B), we plot the subgrid kinetic energy fraction, $\bar{K}_{s g s} /\left(\bar{K}_{\text {res }}+\bar{K}_{s g s}\right)$, where $K_{\text {res }}=\widetilde{u}_{i} \widetilde{u}_{i} / 2$ and $K_{s g s}=T_{i i} / 2=K$, in figure 7 . Near the channel centre, the LES SGS model behaves in the classical LES view, where it carries roughly $10 \%-20 \%$ of the overall kinetic energy. This, however, is reversed near the wall, independent of $R e_{\tau}$ and resolution (compare figures $7 a$ and $7 b$ ).

A feature of our wall model is its ability to predict the local Kármán constant, $\mathscr{K}_{1}$. Its p.d.f. in figure 8 shows peak values around 0.38 , with a wider distribution for the cases with $\gamma_{\text {III }}=0.45$. The notion of variable $\mathscr{K}_{1}$ was also suggested by Nickels (2004), who found that $\mathscr{K}_{1}$ could also be sensitive to pressure gradients. This is consistent with the present modelling approach, where all local wall-adjacent cells are subjected to outer flow forcing with pressure gradients and should therefore have their own $\mathscr{K}_{1}$, although their average $\overline{\mathscr{K}}_{1}$ should agree with experiments or DNS data.

Figure 9 shows that the mean velocity profiles are not very sensitive to a doubling of the virtual wall location, $h_{0}$, from $h_{0}=0.18 \Delta_{z}$ to $h_{0}=0.36 \Delta_{z}$. We 

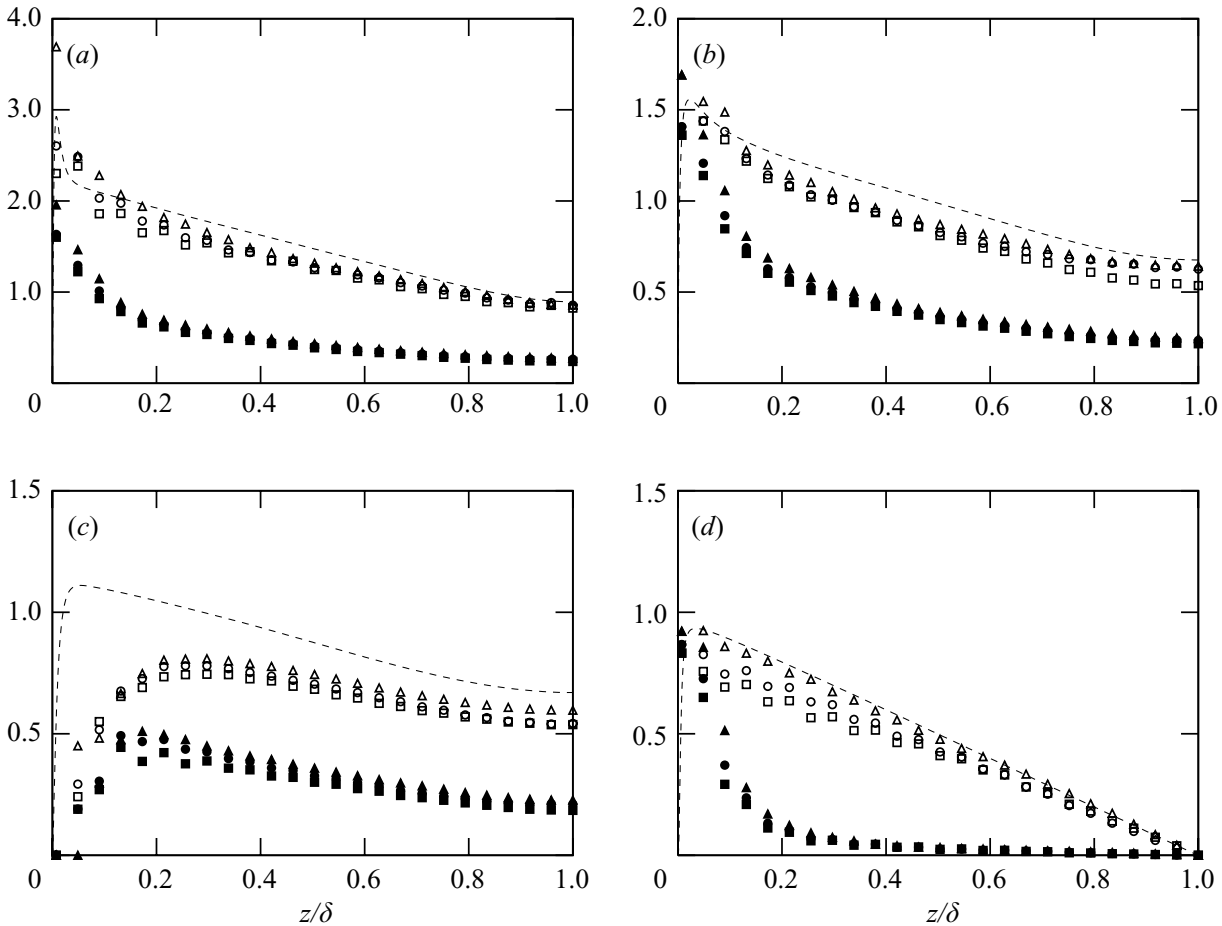

FIGURE 6. Turbulence statistics, (a) $u_{r m s}^{+}$, (b) $v_{r m s}^{+}$, (c) $w_{r m s}^{+}$and $(d)-\overline{u w}^{+}$, for LES with $\gamma_{\mathrm{III}}=0.45(\mathrm{set} \mathrm{C}): \square, R e_{\tau}=20 \mathrm{k} ; \bigcirc, R e_{\tau}=200 \mathrm{k} ; \triangle, R e_{\tau}=2 \mathrm{M} ;----, R e_{\tau}=2 \mathrm{k}$ DNS (Hoyas \& Jiménez 2006): open symbols, total (resolved plus subgrid); solid symbols, subgrid.
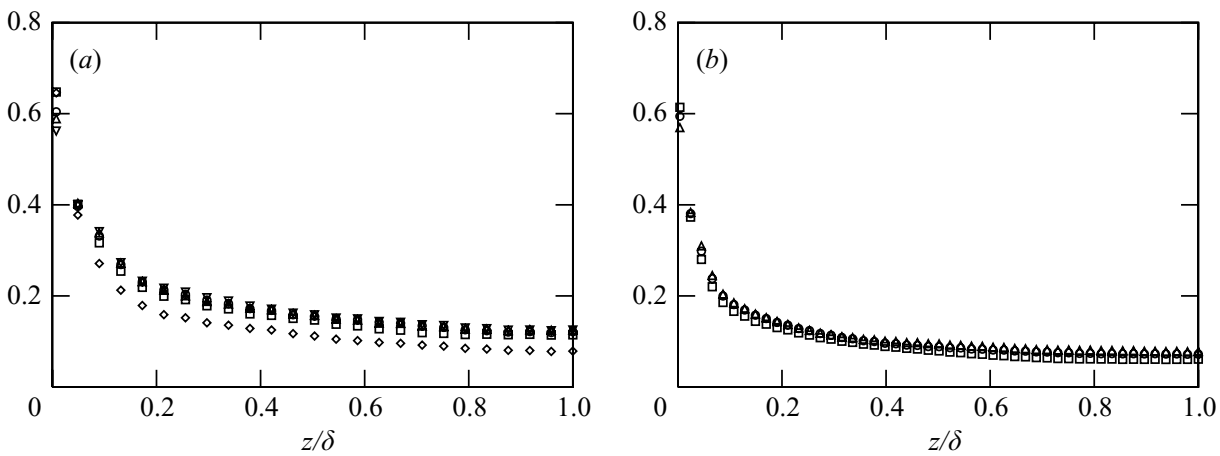

FiguRE 7. The subgrid kinetic energy fraction $\bar{K}_{s g s} /\left(\bar{K}_{\text {res }}+\bar{K}_{s g s}\right)$ with $(a)$ coarse resolution (set A) and (b) fine resolution (set B): $\diamond, R e_{\tau}=2 \mathrm{k} ; \square, R e_{\tau}=20 \mathrm{k} ; \bigcirc, R e_{\tau}=200 \mathrm{k} ; \Delta, R e_{\tau}=2 \mathrm{M}$; $\nabla, R e_{\tau}=20 \mathrm{M}$.

found some sensitivity when $h_{0}$ was decreased below $0.1 \Delta_{z}$, and this is not recommended. With $\zeta_{0} \equiv h_{0} / \Delta_{z}$ and $\Delta_{z}=L_{z} / N_{z}, \delta=L_{z} / 2+h_{0}$, we have, in inner variables, $h_{0}^{+}=2 \zeta_{0} R e_{\tau} /\left(N_{z}+2 \zeta_{0}\right)$. We require that $h_{0}^{+}>h_{v}^{+}=11$. This is satisfied always presently. We note that for $R e_{\tau}=2 \mathrm{k}$, this is not satisfied with $N=96$, and these runs were done only with $N=48$ for which $h_{0}^{+} \approx 15$ with $\zeta_{0}=0.18$. 

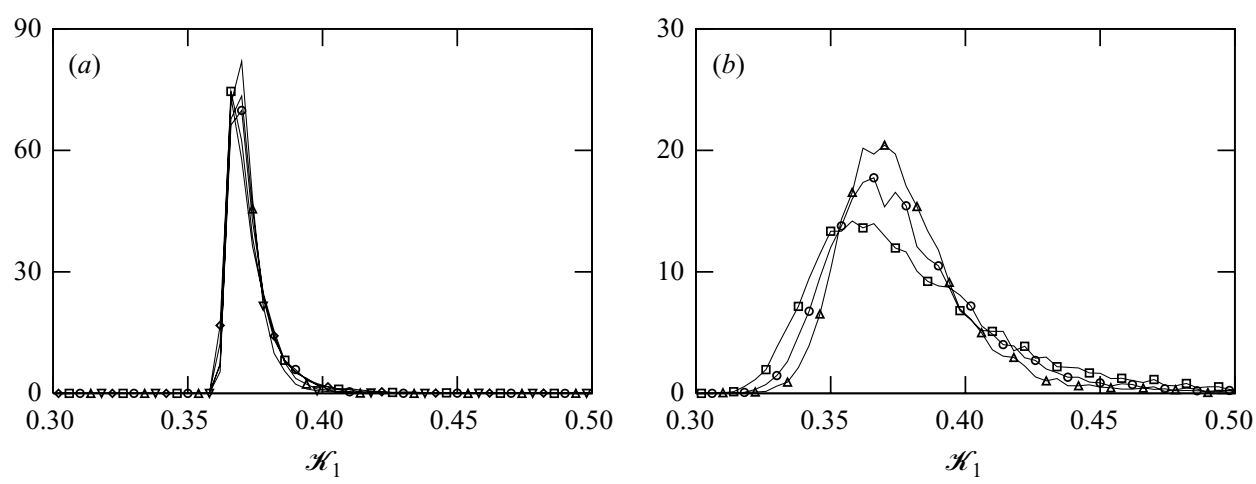

FIGURE 8. P.d.f. of the predicted Kármán constant, $\mathscr{K}_{1}$, for LES with (a) $\gamma_{\mathrm{III}}=0$ (set A), $\left(\overline{\mathscr{K}}_{1}\right)_{\text {avg }}=0.37$ and $(b) \gamma_{\mathrm{III}}=0.45$ (set C), $\left(\overline{\mathscr{K}}_{1}\right)_{\text {avg }}=0.39: \diamond, R e_{\tau}=2 \mathrm{k} ; \square, R e_{\tau}=20 \mathrm{k} ; 0$, $R e_{\tau}=200 \mathrm{k} ; \Delta, R e_{\tau}=2 \mathrm{M} ; \nabla, R e_{\tau}=20 \mathrm{M}$.

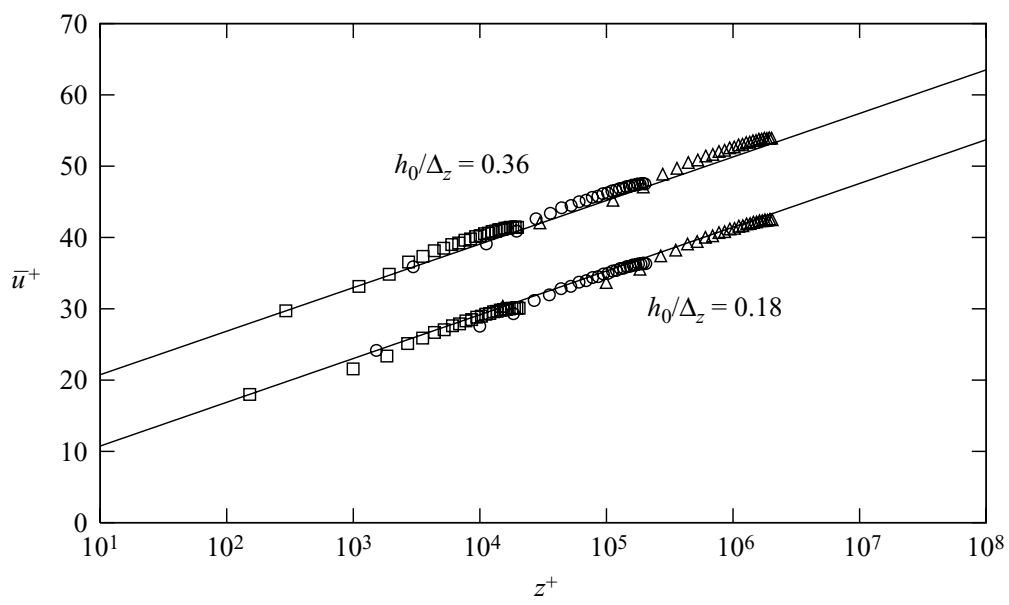

FIGURE 9. Model sensitivity to virtual wall location, $h_{0}$ : upper set (set F), $\left(\overline{\mathscr{K}}_{1}\right)_{\text {avg }}=0.38$, shifted up by 10 units for clarity; lower set (set A), $\left(\overline{\mathscr{K}}_{1}\right)_{\text {avg }}=0.37 ; \square, R e_{\tau}=20 \mathrm{k} ; \bigcirc, R e_{\tau}=$ $200 \mathrm{k} ; \triangle, R e_{\tau}=2 \mathrm{M} ;-, \log \left(z^{+} / 11\right) /\left(\overline{\mathscr{K}}_{1}\right)_{\text {avg }}+11$.

\subsection{Resolved-scale spectra}

The one-dimensional power spectra of streamwise velocity fluctuations, $E_{u u}$, at the quarter-channel height $z / \delta=0.5$ is shown in figure 10 . We adopt the following convention:

$$
u_{\mathrm{rms}}^{2}=\int_{0}^{\infty} E_{u u}\left(k_{x}\right) \mathrm{d} k_{x}=\int_{0}^{\infty} E_{u u}\left(k_{y}\right) \mathrm{d} k_{y} .
$$

The plots are normalized with the plane-averaged Kolmogorov scales $\bar{\epsilon}$ and $\nu$, where the total dissipation $\epsilon=\epsilon_{\text {res }}+\epsilon_{s g s}$, the resolved dissipation $\epsilon_{\text {res }}=2 \nu \widetilde{S}_{i j} \widetilde{S}_{i j}$ and the subgrid dissipation $\epsilon_{s g s}=\int_{k_{c}}^{\infty} 2 v k^{2} E(k) \mathrm{d} k$. For reference, $\bar{K}_{s g s} /\left(\bar{K}_{r e s}+\bar{K}_{s g s}\right) \lesssim 0.2$ at this wall-normal plane (figure 7a). Also in figure 10 are model spectra (Pope 2000, figure 6.14) that fit data from nearly two decades (30-1500) of Taylor-scale Reynolds numbers, as compiled by Saddoughi \& Veeravalli (1994). To reproduce the model spectra of Pope (2000), we use the parameters $\bar{\epsilon}, v$ and the isotropic surrogate for the 


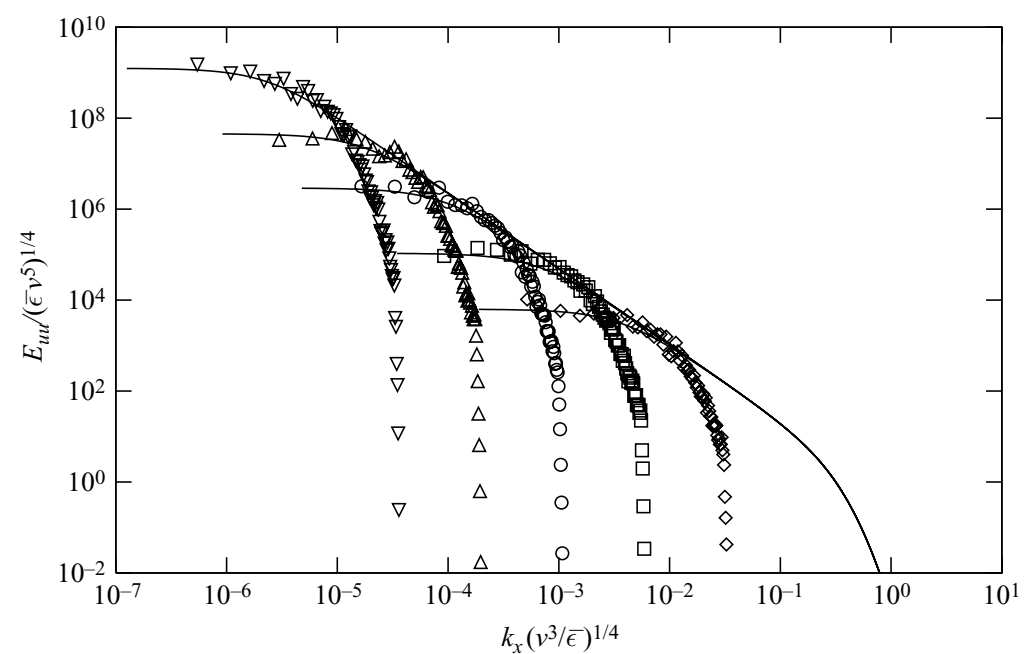

FIGURE 10. Resolved spectra at $z / \delta=0.5$ for set $\mathrm{A}: \diamond, R e_{\tau}=2 \mathrm{k} ; \square, R e_{\tau}=20 \mathrm{k} ; \bigcirc$, $R e_{\tau}=200 \mathrm{k} ; \triangle, R e_{\tau}=2 \mathrm{M} ; \nabla, R e_{\tau}=20 \mathrm{M} ;-$, model spectra (Pope 2000).

turbulent kinetic energy, $3 u_{r m s}^{2} / 2$, where $u_{r m s}^{2}=\overline{\widetilde{u} \widetilde{u}}+\bar{T}_{x x}$. The envelope produced by the composite of all the spectra collapses onto the same $k^{-5 / 3}$ line, suggesting that in all cases, the Kolmogorov scale - and hence the total dissipation - are accurately predicted by the LES model, independent of $R e_{\tau}$. The rapid ( $\approx 6$ points) drop near the 2/3-cutoff wavenumber is purely an artefact of the exponential smoothing, (A 6). The rest of the drop could be attributed to dominant SGS dynamics that overwhelm the resolved scales in that wavenumber range, resulting in the observed effect of excess resolved wavenumbers. To test this idea, we need the subgrid extension of the spectrum representing the dynamics of the SGS model.

\subsection{Subgrid-continued spectra}

A benefit of the stretched-spiral vortex model for LES is the availability of a closed-form spectral representation of the local Navier-Stokes solution that can be used to obtain the subgrid-continued spectra down to Kolmogorov scales. This was demonstrated for both velocity and scalar spectra by Hill et al. (2006).

Given that the SGS vortices are oriented according to the delta-function distribution (§2.2), the one-dimensional spectrum tensors derived by Pullin \& Saffman (1994, equations (49)-(51)) for an ensemble of cylindrical vortices reduce to

$$
\begin{aligned}
\Theta_{11}\left(k_{3}\right)= & \frac{2}{\pi} \int_{\left|k_{3} / \sin \theta\right|}^{\infty} E(k)\left(k^{2}-\frac{k_{3}^{2}}{\sin ^{2} \theta}\right)^{-1 / 2} \\
& \times\left[\frac{1}{k^{2}} \cos ^{2} \theta \cos ^{2} \phi\left(k^{2}-\frac{k_{3}^{2}}{\sin ^{2} \theta}\right)+\frac{1}{k^{2}} \sin ^{2} \phi \frac{k_{3}^{2}}{\sin ^{2} \theta}\right] \frac{1}{\sin \theta} \mathrm{d} k, \\
\Theta_{22}\left(k_{3}\right)= & \frac{2}{\pi} \int_{\left|k_{3} / \sin \theta\right|}^{\infty} E(k)\left(k^{2}-\frac{k_{3}^{2}}{\sin ^{2} \theta}\right)^{-1 / 2} \\
& \times\left[\frac{1}{k^{2}} \cos ^{2} \theta \sin ^{2} \phi\left(k^{2}-\frac{k_{3}^{2}}{\sin ^{2} \theta}\right)+\frac{1}{k^{2}} \cos ^{2} \phi \frac{k_{3}^{2}}{\sin ^{2} \theta}\right] \frac{1}{\sin \theta} \mathrm{d} k,
\end{aligned}
$$



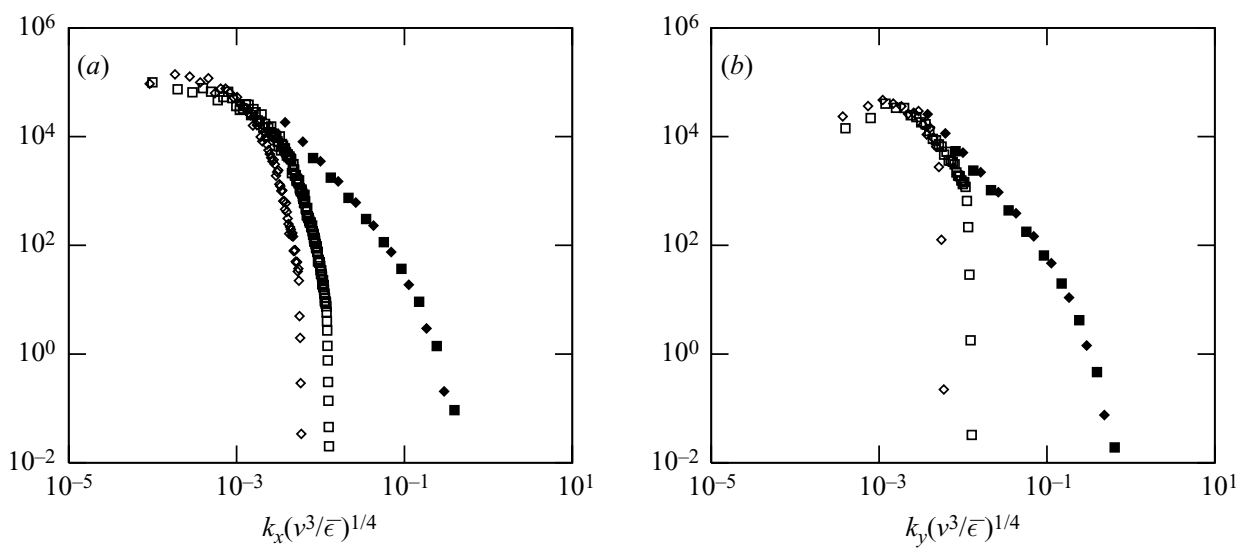

FIGURE 11. Effect of grid resolution on $(a) E_{u u}\left(k_{x}\right) /\left(\bar{\epsilon} v^{5}\right)^{1 / 4}$ and $(b) E_{u u}\left(k_{y}\right) /\left(\bar{\epsilon} v^{5}\right)^{1 / 4}$ at $z / \delta=0.5$ for $R e_{\tau}=20 \mathrm{k}: \diamond$ case A2; $\square$ case B2; open symbols, resolved; solid symbols, subgrid.

$$
\begin{aligned}
\Theta_{33}\left(k_{3}\right)= & \frac{2}{\pi} \int_{\left|k_{3} / \sin \theta\right|}^{\infty} E(k)\left(k^{2}-\frac{k_{3}^{2}}{\sin ^{2} \theta}\right)^{-1 / 2} \\
& {\left[\frac{1}{k^{2}} \sin ^{2} \theta\left(k^{2}-\frac{k_{3}^{2}}{\sin ^{2} \theta}\right)\right] \frac{1}{\sin \theta} \mathrm{d} k, }
\end{aligned}
$$

with $E(k)$ is given by (2.6). Here, the Euler angles $\theta$ and $\phi$ (the delta-function peak locations) are defined relative to the $\left(x_{1}, x_{2}, x_{3}\right)$ coordinate system, which is not necessarily coincident with the laboratory frame (different from $\S 2.2$ ). The even function $\Theta_{i i}\left(k_{3}\right)$, no summation over $i$, is defined such that $\int_{-\infty}^{\infty} \Theta_{i i}\left(k_{3}\right) \mathrm{d} k_{3}=\left(u_{i}\right)_{r m s}^{2}$, from which it follows that $E_{i i}\left(k_{3}\right)=2 \Theta_{i i}\left(k_{3}\right)$. To obtain the subgrid-continued $E_{i i}\left(k_{x}\right)$, we choose $k_{3}$ to coincide with $k_{x}$; that is $\theta$ is the angle between the vortex axis and the $x$-axis. Given the wavenumber $k_{x}, E_{i i}\left(k_{x}\right)$ is found by averaging $(4.2 a)$ over every cell on the $x y$-plane; $E_{i i}\left(k_{y}\right)$ is obtained in a similar manner. We remark that this calculation requires only numerical information obtained from the LES through $E(k)$; no additional tuning parameters are required. Also, this calculation is only performed when subgrid-continued spectra are required; it is not a required part of the resolved-scale LES simulation.

Figure 11 shows both resolved and subgrid contributions to $E_{u u}\left(k_{x}\right)$ and $E_{u u}\left(k_{y}\right)$ for the $R e_{\tau}=20 \mathrm{k}$ case with two different grid resolutions. As the resolution is increased, the resolved spectra extend themselves to higher wavenumbers, following their subgrid extensions. Note, however, that the subgrid extensions remain unchanged as the resolution is increased; on this basis, one could claim that the SGS dynamics have converged. Unlike $E_{u u}\left(k_{x}\right), E_{u u}\left(k_{y}\right)$ appears not to suffer from excess resolution, dropping sharply only near the 2/3-cutoff wavenumber. The subgrid part of $E_{u u}\left(k_{x}\right)$ (see figure 11a) continues where the resolved part drops off, reinforcing the idea that the SGS dynamics effectively take over.

We compare our LES prediction of $E_{u u}$ at various wall distances with the DNS data of Hoyas \& Jiménez (2006) in figure 12. The $z / \delta=0.2$ plane corresponds to our fifth off-wall grid point, where $\bar{K}_{s g s} /\left(\bar{K}_{\text {res }}+\bar{K}_{s g s}\right) \approx 0.2$ (see figure $7 a$ ). At these wall locations, the composite of the resolved and subgrid components appear to faithfully capture the main features of the DNS spectra. These features include the spatial anisotropy of the subgrid extensions, seen in the ordering of the subgrid $E_{u u}\left(k_{x}\right)$ and 

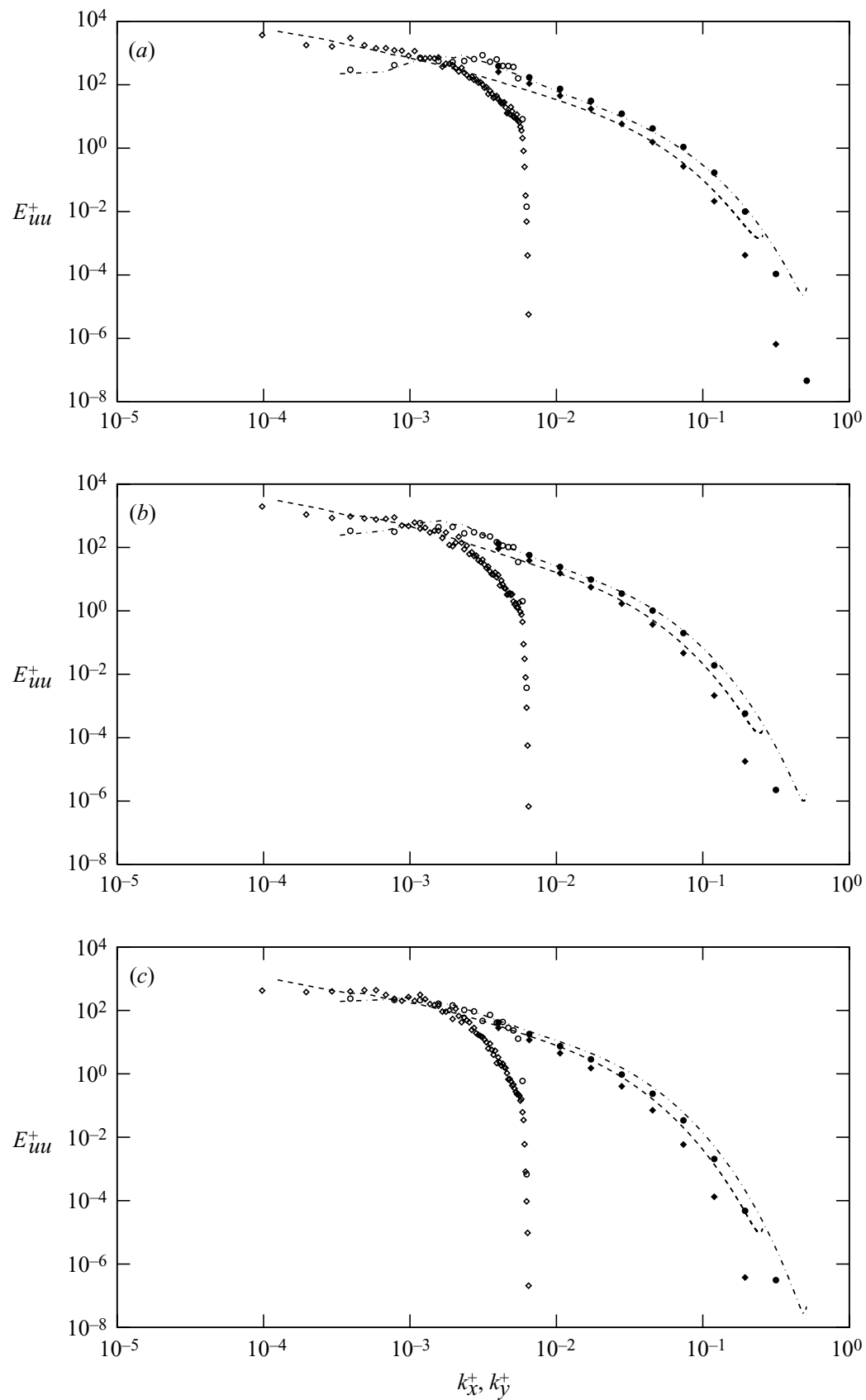

FiguRE 12. Spectra for $R e_{\tau}=2 \mathrm{k}$ (case A1) at $(a) z / \delta=0.2,(b) z / \delta=0.5$ and $(c) z / \delta=1$ : $\diamond$ and ----,$E_{u u}^{+}\left(k_{x}^{+}\right) ; \bigcirc$ and,$--- E_{u u}^{+}\left(k_{y}^{+}\right)$; open symbols, resolved; solid symbols, subgrid; lines, DNS (Hoyas \& Jiménez 2006).

$E_{u u}\left(k_{y}\right)$, and the hump in the resolved $E_{u u}\left(k_{y}\right)$ in the range $10^{-3}<k_{y}^{+}<10^{-2}$, which becomes less prominent as $z / \delta$ increases from 0.2 to 1 . In figure $12(a)$, the subgrid extension of $E_{u u}\left(k_{x}\right)$ is slightly steeper than the DNS result. This may be due to a mismatch between the near-wall physics, which, perhaps, is exhibiting a $k^{-1}$ range 

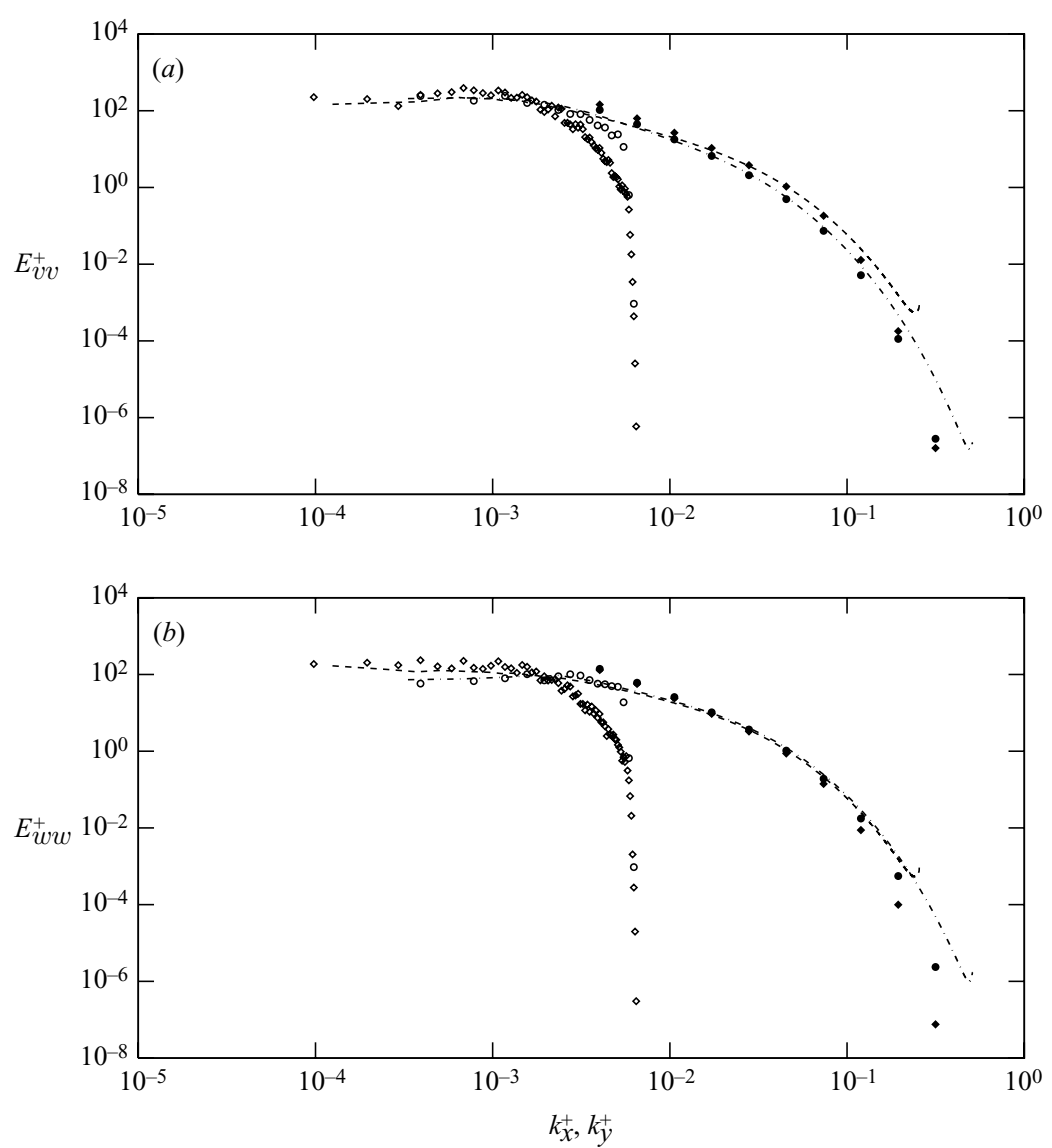

FIGURE 13. Spectra for $R e_{\tau}=2 \mathrm{k}$ (case A1) at $z / \delta=0.5: \diamond$ and,$---- E_{v v}^{+}\left(k_{x}^{+}\right)$and $E_{w w}^{+}\left(k_{x}^{+}\right)$; $\bigcirc$ and,$--- E_{v v}^{+}\left(k_{y}^{+}\right)$and $E_{w w}^{+}\left(k_{y}^{+}\right)$; open symbols, resolved; solid symbols, subgrid; lines, DNS (Hoyas \& Jiménez 2006).

(Perry \& Chong 1982), and our LES model based on stretched-spiral vortices, which have an inherent $k^{-5 / 3}$ inertial range.

We also plot one-dimensional spectra for the spanwise and wall-normal velocity components at $z / \delta=0.5$ in figure 13. Comparing the spectra for the three velocities at the same wall-normal location (figures $13 a, b$ and $12 b$ ), we see a faithful reproduction of the velocity anisotropy, as well as the spatial anisotropy, both in the resolved components and the subgrid extension; in fact, the ordering of the subgrid $E_{u u}\left(k_{x}\right)$ and $E_{u u}\left(k_{y}\right)$ is switched for the subgrid $E_{v v}\left(k_{x}\right)$ and $E_{v v}\left(k_{y}\right)$ in $10^{-2}<k_{x}^{+}, k_{y}^{+}<10^{-1}$.

\section{Conclusions}

An LES wall model based on SGS stretched vortices has been developed. Its salient feature is the implementation of Dirichlet boundary conditions, including a streamwise slip velocity at a lifted, virtual wall that lies within the overlap region. This is done using a tailored SGS near-wall model based on the plane filtering and wallnormal averaging of the streamwise momentum equation combined with an extended version of the general stretched-vortex SGS model that incorporates the dominant near-wall physics. When coupled with outer, resolved-scale LES, use of the wall model 
produces satisfactory mean velocity profiles and acceptable second-order turbulence statistics over a wide range of $R e_{\tau}$. This scheme also provides dynamic estimates of the Kármán constant that are consistent with experimental measurements. In the main body of the channel flow, subgrid-continued spectra agree well with DNS down to Kolmogorov scales and capture anisotropy effects.

Since the present near-wall model is entirely local, it can readily be applied to inhomogeneous turbulent flows. While the predictive performance of a wall model in complex turbulent flows, especially those involving separation, can only be validated with an a posteriori analysis and further LES, some prior analysis may guide our expectations of the present model, which we now discuss.

First, we have argued that our approach is not an equilibrium model in the sense of Piomelli (2008). Presently the local inner-scaling assumption (3.6) is used in a local sense to reduce only the unsteady term in the plane-filtered, wall-normal averaged streamwise momentum equation, leading to (3.12). We stress again that in this reduction, a specific $F\left(z^{+}\right)$is not required. Further, our log-like profile (3.19) does not use (3.6) but is derived directly from the stretched-vortex subgrid model combined with the attached eddy hypothesis in a way that couples with the resolvedscale LES. In fact, the effective and local 'Kármán constant' (3.18) depends partially on LES-derived quantities and so is also a mixed inner-outer scale parameter. Thus the combination of (3.12) and (3.19) can be viewed as containing elements of both restricted inner scaling and the outer flow via the LES. The overall model is therefore only partially reliant upon inner scaling. This may be an improvement over TBLE approaches, where the log law is implied by the damped mixing-length eddy viscosity. We note that even when this log law is assumed, the TBLE approach improves the prediction of separated flows relative to equilibrium log-law models (Cabot \& Moin 1999).

Second, we can show that (3.12) contains an inherent signature for incipient separation in the sense that $u_{\tau}(x, y, t)$ vanishes locally. At each wall-adjacent $(x, y)$ location, (3.12) can be written in the form $\left(\eta_{0} \equiv \partial \widetilde{u} /\left.\partial z\right|_{0}\right)$

$$
\frac{\mathrm{d} \eta_{0}}{\mathrm{~d} t}=\Lambda \eta_{0}\left(\widetilde{\eta}_{0}-\eta_{0}\right)
$$

where

$$
\Lambda(t)=\frac{2 v}{\left.h \widetilde{u}\right|_{h}}, \quad \widetilde{\eta}_{0}(t)=-\left.\frac{1}{v} \widetilde{u w}\right|_{h}-\frac{h}{v}\left(\frac{\left.\partial \widetilde{u u}\right|_{h}}{\partial x}+\frac{\left.\partial \widetilde{u v}\right|_{h}}{\partial y}+\left.\frac{\partial \widetilde{p}}{\partial x}\right|_{h}\right)+\left.\frac{\partial \widetilde{u}}{\partial z}\right|_{h} .
$$

After using the integrating factor,

$$
I(t)=\exp \left(-\int_{0}^{t} \Lambda(s) \widetilde{\eta}_{0}(s) \mathrm{d} s\right)
$$

(5.1) becomes separable, and its exact solution, written as an integral, is

$$
\frac{1}{\eta_{0}(t)}=\frac{I(t)}{\eta_{0}(0)}+I(t) \int_{0}^{t} \frac{\Lambda(s)}{I(s)} \mathrm{d} s
$$

For simplicity now assume constant coefficients $\widetilde{\eta}_{0}(t)=\widetilde{\eta}_{0}(0)$ and $\Lambda(t)=\Lambda(0)$; then

$$
\frac{1}{\eta_{0}(t)}=\frac{1}{\eta_{0}(0)} \mathrm{e}^{-t \Lambda(0) \widetilde{\eta}_{0}(0)}+\frac{1}{\widetilde{\eta}_{0}(0)}\left(1-\mathrm{e}^{-t \Lambda(0) \widetilde{\eta}_{0}(0)}\right)
$$

so that $\eta_{0}(t) \sim \widetilde{\eta}_{0}(0)$ for $t \gg 1 /\left[\Lambda(0) \widetilde{\eta}_{0}(0)\right]$. Roughly, this means $\eta_{0}$ tends towards the steady state $\widetilde{\eta}_{0}$ at the rate $\Lambda \widetilde{\eta}_{0}$. A separation criterion can now be obtained based 
on (5.4). Without loss of generality, assume that $\eta_{0}(t=0)>0$. Separation, defined by $\eta_{0}=0$, would occur at $t=t_{s}>0$. We know $I\left(t_{s}\right)>0$ from (5.3), and upon assuming that $I\left(t_{s}\right)<\infty$, we require the integral in (5.4) to be singular for separation. A sufficient condition is $\Lambda(t)=O\left(t-t_{s}\right)^{-1}$ to give a logarithmic singularity. From the definition of $\Lambda(t)$ in $(5.2 a)$, we require that $\left.\widetilde{u}\right|_{h}=O\left(t-t_{s}\right)$. When $t>t_{s},\left.\widetilde{u}\right|_{h}$ changes sign, indicating that the outer flow has separated and is now reversed.

When the lifted wall penetrates the outer edge of the viscous sublayer, $h_{0}^{+}<h_{v}^{+}=11$, which occurs near separation or in a laminar flow, the logarithmic boundary condition (3.19) would be replaced with the linear relationship $\left.\widetilde{u}^{+}\right|_{h_{0}}=h_{0}{ }^{+}$.

Finally, in the interests of minimizing discretization errors, we tested the present model on a purpose-built high-accuracy research code. However, we expect similar model performance on general-purpose codes - see Pantano et al. (2008), who successfully employed a second-order code to run a similar LES SGS model coupled with a different wall model.

The authors wish to thank Professor J. Jiménez who kindly provided DNS spectra. This work is partially supported by the NSF under grant CBET 0651754.

\section{Appendix A. Numerical method}

The low-storage third-order semi-implicit Runge-Kutta method of Spalart, Moser \& Rogers (1991) is used for temporal discretization. The implicit treatment of the viscous term allows large time steps to be taken; in the simulations, the CFL number

$$
\Delta_{t} \max \left(\frac{|u|}{\Delta_{x}}, \frac{|v|}{\Delta_{y}}, \frac{|w|}{\Delta_{z}}\right)
$$

was set to unity. The low-storage property simplifies the time step advancement into three sequential substeps, $n=0,1,2$, of the same form:

$$
\begin{aligned}
\frac{\widetilde{u}_{i}^{(n+1)}-\widetilde{u}_{i}^{(n)}}{\Delta_{t}} & =-\left(\gamma_{n}+\zeta_{n}\right) \frac{\partial \widetilde{p}^{(n+1)}}{\partial x_{i}}+\gamma_{n} H_{i}^{(n)}+\zeta_{n} H_{i}^{(n-1)}+\alpha_{n} L_{i}^{(n)}+\beta_{n} L_{i}^{(n+1)}, \\
\frac{\partial \widetilde{u}_{i}^{(n+1)}}{\partial x_{i}} & =0, \\
H_{i} & =-\frac{1}{2}\left(\frac{\partial \widetilde{u}_{i} \widetilde{u}_{j}}{\partial x_{j}}+\widetilde{u}_{j} \frac{\partial \widetilde{u}_{i}}{\partial x_{j}}\right)+\frac{\partial T_{i j}}{\partial x_{j}}+f_{i}, \quad L_{i}=v \frac{\partial^{2} \widetilde{u}_{i}}{\partial x_{j}^{2}} .
\end{aligned}
$$

For an arbitrary reference time $t_{0}, \widetilde{u}_{i}^{(3)}=\widetilde{u}_{i}\left(t_{0}+\Delta_{t}\right)$. The value of the constants $\alpha_{n}, \beta_{n}$, $\gamma_{n}$ and $\zeta_{n}$ are given by Spalart et al. (1991). The discrete pressure $\widetilde{p}^{(n+1)}$ is formally a Lagrange multiplier that enforces (A $2 b$ ), including at the boundary.

The governing equations are solved in an $L_{x} \times L_{y} \times L_{z}$ box with periodic boundary conditions applied in both the $x$ and $y$ directions. Applying the Fourier spectral method for both these directions results in $N_{x} \times N_{y}$ sets of one-dimensional complex Helmholtz equations in the $z$ direction for each mode $\left(k_{x}, k_{y}\right)$, where

$$
\begin{aligned}
& k_{x}=2 \pi / L_{x}\left(-N_{x} / 2, \ldots,-1,0,1, \ldots, N_{x} / 2-1\right), \\
& k_{y}=2 \pi / L_{y}\left(-N_{y} / 2, \ldots,-1,0,1, \ldots, N_{y} / 2-1\right) .
\end{aligned}
$$

In practice, only half of the $N_{x} \times N_{y}$ sets of complex equations (or $N_{x} \times N_{y}$ sets of real equations, separately counting real and imaginary parts) are solved due to the symmetry of the Fourier coefficients. 
An explicit finite-difference scheme with fourth-order global accuracy (thirdorder boundary, sixth-order interior) is used to approximate the $z$ derivative on $N_{z}+1$ uniformly spaced nodes, located at $z_{k}=L_{z}\left(-1 / 2+k / N_{z}\right), k=0,1, \ldots, N_{z}$. When coupled with a certain diagonal norm, the finite-difference approximation satisfies the summation-by-parts (SBP) property (Carpenter, Gottlieb \& Abarbanel 1994; Strand 1994; Mattsson \& Nordström 2004). Although stability in the sense described in those papers is not preserved because Dirichlet boundary conditions are applied directly (injection method), our experience indicates that stability is not an issue, at least presently, where the diffusion operator is advanced implicitly. In explicit schemes, a seven-point stencil is required to achieve sixth-order accuracy, but, following Hill \& Pullin (2004), we use two additional points to minimize the Ghosal truncation error (Ghosal 1996). The Ghosal truncation error pertains to errors resulting from the application of spatial discretization schemes to the Navier-Stokes equations. Unsurprisingly, in reducing the Ghosal truncation error, the high-wavenumber performance of the finite-difference scheme is also simultaneously improved (Hill \& Pullin 2004). Such a scheme, called a tuned centred-difference (TCD) method by Hill \& Pullin (2004), is also combined with the SBP scheme in Pantano et al. (2007). Complete details of the $N_{z}+1 \times N_{z}+1$ finite-difference matrix $D_{z}$ are provided in Appendix B.

Spatially discretizing (A 2), we obtain for each mode $\left(k_{x}, k_{y}\right)$ and node $z_{k}$

$$
\left.\begin{array}{c}
\left(D_{z}^{2}-\lambda^{2}\right) \widehat{u}=\mathrm{i} k_{x} \zeta^{\prime} \widehat{p}+R_{1}+\sigma_{1}, \\
\left(D_{z}^{2}-\lambda^{2}\right) \widehat{v}=\mathrm{i} k_{y} \zeta^{\prime} \widehat{p}+R_{2}+\sigma_{2}, \\
\left(D_{z}^{2}-\lambda^{2}\right) \widehat{w}=D_{z} \zeta^{\prime} \widehat{p}+R_{3}+\sigma_{3}, \\
\mathrm{i} k_{x} \widehat{u}+\mathrm{i} k_{y} \widehat{v}+D_{z} \widehat{w}=0,
\end{array}\right\}
$$

where $\lambda^{2}=k_{x}^{2}+k_{y}^{2}+1 /\left(\beta v \Delta_{t}\right)$ and $\zeta^{\prime}=(\gamma+\zeta) /(\beta v)$. The spatial discretization of $\left(u_{1}, u_{2}, u_{3}, p\right)^{(n+1)}(x, y, z)$ is denoted $(\widehat{u}, \widehat{v}, \widehat{w}, \widehat{p})\left(k_{x}, k_{y}, k\right)$. For clarity, we have dropped the superscript and subscript $n$ from (A $2 a) ; R_{i}$ are known terms, obtained by simple rearrangement; $\sigma_{i}$ are incurred penalty terms for directly imposing Dirichlet boundary conditions on the velocity at $k=0$ and $k=N_{z}$ (or equivalently, at $z= \pm L_{z} / 2$ ),

$$
\left.\begin{array}{rl}
(\widehat{u}, \widehat{v}, \widehat{w})\left(k_{x}, k_{y}, 0\right) & =\left({\widehat{g_{1}}}^{-},{\widehat{g_{2}}}^{-},{\widehat{g_{3}}}^{-}\right)\left(k_{x}, k_{y}\right), \\
(\widehat{u}, \widehat{v}, \widehat{w})\left(k_{x}, k_{y}, N_{z}\right) & =\left({\widehat{g_{1}}}^{+},{\widehat{g_{2}}}^{+},{\widehat{g_{3}}}^{+}\right)\left(k_{x}, k_{y}\right) . .
\end{array}\right\}
$$

In other words, because we choose to satisfy (A 4), (A 3) written without $\sigma_{i}$ cannot be satisfied at $k=0$ and $k=N_{z}$. Although we call $\sigma_{i}$ a penalty term, it should not be confused with the simultaneous-approximation-term (SAT) technique (Carpenter et al. 1994), where $\sigma_{i}$ is explicitly specified. Here, $\sigma_{i}$ must be determined as part of the solution procedure. Note, however, that the divergence constraint, (A $3 b$ ), is satisfied everywhere, even at the boundaries, $k=0$ and $k=N_{z}$.

The discrete pressure Poisson equation is obtained by applying the discrete divergence to the momentum equations:

$$
\left(D_{z}^{2}-\left[k_{x}^{2}+k_{y}^{2}\right]\right) \zeta^{\prime} \hat{p}=-R_{p}-\left(\mathrm{i} k_{x} \sigma_{1}+\mathrm{i} k_{y} \sigma_{2}+D_{z} \sigma_{3}\right)
$$

where $R_{p}=\mathrm{i} k_{x} R_{1}+\mathrm{i} k_{y} R_{2}+D_{z} R_{3}$. In general, $D_{z} \sigma_{3}$ is non-zero in the interior of the domain, $k=1,2, \ldots, N_{z}-1$. That is $\sigma_{i}$, appearing only at the boundaries of (A $3 a$ ) carry over as additional interior source terms to (A 5). Careful accounting of the $\sigma_{i}$ terms is key to ensuring discrete mass conservation up to machine precision. Unlike in well-resolved simulations such as DNS in which $\sigma_{i}$ is small and can thus be safely 
ignored, $\sigma_{i}$ in LES is large and if ignored will be a source of non-zero velocity divergence that leads to numerical instability (Canuto et al. 1987). The method for solving this coupled problem in the discrete framework is the Kleiser-Schumann influence-matrix method, detailed by Canuto et al. (1987). Although they presented it for the Chebyshev-tau and spectral collocation discretizations with homogeneous boundary conditions, the method is quite general, and we extend its usage here for finite differences with non-homogeneous boundary conditions.

As written, the nonlinear products in (A $2 c$ ) are computed in skew-symmetric form in physical space. Then, $H_{i}$, which includes the nonlinear products and the LES contributions, is transformed to wavenumber space, where a $p_{1}$ th-order Fourier exponential filter (Gottlieb \& Shu 1997; Hou \& Li 2007) of the form

$$
\sigma(\xi)=\exp \left(-\alpha_{1} \xi^{p_{1}}\right)
$$

is applied in the periodic directions, $\xi=\left|k_{x}\right| /\left(\pi / \Delta_{x}\right),\left|k_{y}\right| /\left(\pi / \Delta_{y}\right) ; p_{1}=36 ; \alpha_{1}$ is chosen so that $\sigma(2 / 3)=1 / 2$. The high-order filter, with flat response at resolved wavenumbers and sharp but smooth drop at the cutoff wavenumber, mimics the 2/3-dealiasing rule. In an LES employing a local physical-space SGS model, the filter's smooth cutoff minimizes Gibbs oscillations that, posing as steep local gradients, would artificially activate the SGS model.

The set of ODEs, (3.12) for the wall shear stress, $\tau_{0}(x, y, t)=v \eta_{0}(x, y, t)$, are advanced using the same third-order Runge-Kutta scheme as the main part of the flow simulation. The overall computing overhead for the implementation of the stretched-vortex SGS model is less than $10 \%$ of the overall computing time. Because the numerical solution of the wall ODE set occurs only in boundary cells, this constitutes a small part of the SGS-related computing effort.

\section{Appendix B. SBP TCD derivative matrix}

We provide details of the fourth-order globally accurate explicit finite-difference $N_{z}+1 \times N_{z}+1$ matrix, $D_{z}$. This matrix operates on any $N_{z}+1$ vector, $u$, say, whose elements $u_{k}$ represent nodal discretizations of the continuous function $u(z)$ at uniformly spaced nodes, $z_{k}=-L_{z} / 2+k \Delta_{z}, \Delta_{z}=L_{z} / N_{z} k=0,1, \ldots, N_{z}$, such that $u\left(z_{k}\right)=u_{k}$. SBP operators (Strand 1994; Mattsson \& Nordström 2004) are constructed from the following decomposition:

$$
D_{z}=\frac{1}{\Delta_{z}} H^{-1} Q, \quad Q+Q^{T}=B, \quad B=\operatorname{diag}(-1,0, \ldots, 0,1) ; \quad(\mathrm{B} 1 a, b, c)
$$

$H=H^{T}>0$ and is used to define an inner product, $(u, u)_{H}=u^{T} H u$, which, in turn, defines a norm on $u$, given by $\|u\|_{H}^{2}=(u, u)_{H}$. Although many possible choices for such $H$ exist, we will only consider the diagonal norm,

$$
H=\operatorname{diag}\left(h_{0}, h_{1}, \ldots, h_{N_{z}}\right),
$$

which restricts the order of accuracy of the boundary scheme to at most half the order of accuracy of the interior scheme (Strand 1994). Although not pursued presently, an SBP scheme with diagonal norm can be easily generalized to apply on arbitrarily mapped grids by absorbing the scaling factors into $H$. Presently, a third-order scheme coupled with a sixth-order interior scheme, equivalent to a fourth-order global scheme, is chosen. The boundary scheme covers the finite-difference approximation for the first and last six points, $k=0,1, \ldots 5, N_{z}-5, N_{z}-4, \ldots, N_{z}$, while the interior scheme covers the rest, $k=6,7, \ldots, N_{z}-6$. 
The sixth-order TCD (Hill \& Pullin 2004), which has a wider nine-point stencil, is used as the interior scheme. The extra degree of freedom is used to improve spectral resolution by reducing truncation errors inherent in the Navier-Stokes equations. Any nine-point sixth-order centred difference approximation for the derivative is given by

$$
D_{z} u_{k}=\frac{1}{\Delta_{z}} \sum_{j=0}^{4} d_{j}\left(u_{k+j}-u_{k-j}\right),
$$

where $d_{0}=0 ; d_{1}=3 / 4-14 d_{4} ; d_{2}=-3 / 20+14 d_{4} ; d_{3}=1 / 60-6 d_{4} ;$ and $d_{4}$ is the free parameter. Setting $d_{4}=0$ recovers the standard sixth-order explicit scheme; setting $d_{4}=-1 / 280$ recovers the standard eighth-order explicit scheme; $d_{4}$ is determined by minimizing the Ghosal truncation error (Ghosal 1996), a procedure detailed by Hill \& Pullin (2004). Here, we only give the final result: $d_{4}=-0.0166$.

Comparing (B 1a), (B 2) and (B 3), we identify the TCD scheme in the context of the SBP operator as

$$
h_{k}=1, \quad Q_{k j}= \begin{cases}d_{j-k}, & 0 \leqslant j-k \leqslant 4, \\ -d_{k-j}, & 0<k-j \leqslant 4, \\ 0, & \text { otherwise }\end{cases}
$$

for $k=6,7, \ldots, N_{z}-6$.

To determine the boundary scheme, write (B 1a) and (B2) explicitly for $k=0,1, \ldots, 5$ :

$$
h_{k}\left(\Delta_{z}\right)\left(D_{z} u\right)_{k}=\sum_{j=0}^{10} Q_{k j} u_{j}=\sum_{j=0}^{5} Q_{k j} u_{j}+\sum_{j=6}^{10} Q_{k j} u_{j}
$$

The second term on the right-hand side is known from using (B $1 b)$ and (4):

$$
Q_{k j}=-Q_{j k}=-\left(-d_{j-k}\right)=d_{j-k}
$$

for $k=0,1, \ldots, 5, j=6,7, \ldots, 10,0<j-k \leqslant 4$. Substituting this in (B 5) gives

$$
h_{k}\left(\Delta_{z}\right)\left(D_{z} u\right)_{k}=\sum_{j=0}^{5} Q_{k j} u_{j}+\sum_{j=6}^{k+4} d_{j-k} u_{j}
$$

The remaining unknowns to be solved are the $6 \times 6$ block, $Q_{k j}(k, j=0,1, \ldots, 5)$ and the six-element vector $h_{k}(k=0,1, \ldots, 5)$. For each $k$, match the coefficients from the series expansion of (B 7) up to $\left(\Delta_{z}\right)^{3}$ and apply (B 1b) to determine the remaining unknowns up to a another free parameter, $Q_{45}$, say, different from $d_{4}$ (see Strand 1994). We choose $Q_{45}$ by minimizing the so-called average boundary truncation error (Diener et al. 2007), giving $Q_{45}=\left(17171-329670 d_{4}\right) / 24300$.

\section{REFERENCES}

Adrian, R. J. 2007 Hairpin vortex organization in wall turbulence. Phys. Fluids 19, 041301.

CАвот, W. \& Morn, P. 1999 Approximate wall boundary conditions in the large-eddy simulation of high Reynolds number flow. Flow Turbul. Combust. 63, 269-291. 
Canuto, C., Hussaini, M. Y., Quarteroni, A. \& Zang, T. A. 1987 Spectral Methods in Fluid Dynamics. Springer.

Carpenter, M. H., Gottlieb, D. \& Abarbanel, S. 1994 Time-stable boundary conditions for finitedifference schemes solving hyperbolic systems: methodology and application to high-order compact schemes. J. Comput. Phys. 111, 220-236.

DeARDorfF, J. W. 1970 A numerical study of three-dimensional turbulent channel flow at large Reynolds numbers. J. Fluid Mech. 41, 453-480.

DeGraafF, D. B. \& Eaton, J. K. 2000 Reynolds-number scaling of the flat-plate turbulent boundary layer. J. Fluid Mech. 422, 319-346.

Del Álamo, J. C., Jiménez, J., Zandonade, P. \& Moser, R. D. 2004 Scaling of the energy spectra of turbulent channels. J. Fluid Mech. 500, 135-144.

Diener, P., Dorband, E. N., Schnetter, E. \& Tiglio, M. 2007 Optimized high-order derivative and dissipation operators satisfying summation by parts, and applications in three-dimensional multi-block evolutions. J. Sci. Comput. 32, 109-145.

Ghosal, S. 1996 An analysis of numerical errors in large-eddy simulations of turbulence. J. Comput. Phys. 125, 187-206.

GotTlieb, D. \& SHU, C. 1997 On the Gibbs phenomenon and its resolution. SIAM Rev. 39, 644-668.

Head, M. R. \& Bandyopadhyay, P. 1981 New aspects of turbulent boundary-layer structure. J. Fluid Mech. 107, 297-338.

Hill, D. J., Pantano, C. \& Pullin, D. I. 2006 Large-eddy simulation and multiscale modelling of a Richtmyer-Meshkov instability with reshock. J. Fluid Mech. 557, 29-61.

Hill, D. J. \& Pullin, D. I. 2004 Hybrid tuned centre-difference - WENO method for large eddy simulations in the presence of strong shocks. J. Comput. Phys. 194, 435-450.

Hou, T. Y. \& LI, R. 2007 Computing nearly singular solutions using pseudo-spectral methods. J. Comput. Phys. 226, 379-397.

Hoyas, S. \& JimÉnEZ, J. 2006 Scaling of the velocity fluctuations in turbulent channels up to $R e_{\tau}=2003$. Phys. Fluids 18, 011702.

Hutchins, N. \& Marusic, I. 2007 Large-scale influences in near-wall turbulence. Phil. Trans. R. Soc. A 365, 647-664.

JimÉNEZ, J. \& Morn, P. 1991 The minimal flow unit in near-wall turbulence. J. Fluid Mech. 225, 213-240.

Lundgren, T. S. 1982 Strained spiral vortex model for turbulent fine structure. Phys. Fluids 25, 2193-2203.

Maruší́, I. \& Perry, A. E. 1995 A wall-wake model for the turbulence structure of boundary layers. Part 2. Further experimental support. J. Fluid Mech. 298, 389-407.

Mattsson, K. \& Nordström, J. 2004 Summation by parts operators for finite difference approximations of second derivatives. J. Comput. Phys. 199, 503-540.

MisRa, A. \& Pullin, D. I. 1997 A vortex-based subgrid stress model for large-eddy simulation. Phys. Fluids 9, 2443-2454.

Nakayama, A., Noda, H. \& Maeda, K. 2004 Similarity of instantaneous and filtered velocity fields in the near wall region of zero-pressure gradient boundary layer. Fluid Dyn. Res. 35, 299-321.

NickeLs, T. B. 2004 Inner scaling for wall-bounded flows subject to large pressure gradients. J. Fluid Mech. 521, 217-239.

Nickels, T. B., Marusic, I., Hafez, S., Hutchins, N. \& Chong, M. S. 2007 Some predictions of the attached eddy model for a high Reynolds number boundary layer. Phil. Trans. R. Soc. A $\mathbf{3 6 5}, 807-822$.

O'Gorman, P. A. \& Pullin, D. I. 2003 The velocity-scalar cross spectrum of stretched spiral vortices. Phys. Fluids 15, 280-291.

Pantano, C., Deiterding, R., Hill, D. J. \& Pullin, D. I. 2007 A low numerical dissipation patchbased adaptive mesh refinement method for large-eddy simulation of compressible flows. J. Comput. Phys. 221, 63-87.

Pantano, C., Pullin, D. I., Dimotakis, P. E. \& Matheou, G. 2008 LES approach for high Reynolds number wall-bounded flows with application to turbulent channel flow. J. Comput. Phys. 227, 9271-9291.

Perry, A. E. \& Chong, M. S. 1982 On the mechanism of wall turbulence. J. Fluid Mech. 119, $173-217$.

Piomelli, U. 2008 Wall-layer models for large-eddy simulation. Prog. Aerosp. Sci. 44, 437-446. 
Piomelli, U. \& Balaras, E. 2002 Wall-layer models for large-eddy simulations. Annu. Rev. Fluid Mech. 34, 349-374.

Pope, S. B. 2000 Turbulent Flows. Cambridge University Press.

Pope, S. B. 2004 Ten questions concerning the large-eddy simulation of turbulent flows. New J. Phys. 6, 35 .

Pullin, D. I. 2000 A vortex-based model for the subgrid flux of a passive scalar. Phys. Fluids 12, 2311-2319.

Pullin, D. I. \& Lundgren, T. S. 2001 Axial motion and scalar transport in stretched spiral vortices. Phys. Fluids 13, 2553-2563.

Pullin, D. I. \& SAfFman, P. G. 1993 On the Lundgren-Townsend model of turbulent fine scales. Phys. Fluids A 5, 126-145.

Pullin, D. I. \& Saffman, P. G. 1994 Reynolds stresses and one-dimensional spectra for a vortex model of homogeneous anisotropic turbulence. Phys. Fluids 6, 1787-1796.

Robinson, S. K. 1991 Coherent motions in the turbulent boundary layer. Annu. Rev. Fluid Mech. 23, 601-639.

Saddoughi, S. G. \& Veeravalli, S. V. 1994 Local isotropy in turbulent boundary layers at high Reynolds number. J. Fluid Mech. 268, 333-372.

Smagorinsky, J. 1963 General circulation experiments with the primitive equations. Part 1. The basic experiment. Mon. Weather Rev. 91, 99-164.

Spalart, P. R., Moser, R. D. \& Rogers, M. M. 1991 Spectral methods for the Navier-Stokes equations with one infinite and two periodic directions. J. Comput. Phys. 96, 297-324.

Strand, B. 1994 Summation by parts for finite difference approximations for d/dx. J. Comput. Phys. $110,47-67$.

Templeton, J. A., Medic, G. \& Kalitzin, G. 2005 An eddy-viscosity based near-wall treatment for coarse grid large-eddy simulation. Phys. Fluids 17, 105101.

Townsend, A. A. 1976 The Structure of Turbulent Shear Flow, 2nd edn. Cambridge University Press.

Voelkl, T., Pullin, D. I. \& Chan, D. C. 2000 A physical-space version of the stretched-vortex subgrid-stress model for large-eddy simulation. Phys. Fluids 12, 1810-1825.

WANG, M. \& Morn, P. 2002 Dynamic wall modelling for large-eddy simulation of complex turbulent flows. Phys. Fluids 14, 2043-2051. 